\title{
Direct Combustion Noise Simulation of a Lean Premixed Swirl Flame using Stochastic Sound Sources
}

\author{
F. Grimm*, D. Ohno ${ }^{\dagger}$, S. Werner ${ }^{\ddagger}$, M. Stöhr ${ }^{\S}$, B. Noll, M. Aigner" \\ Institute of Combustion Technology \\ German Aerospace Center (DLR) \\ Pfaffenwaldring 38-40, 70569 Stuttgart, Germany
}

R. Ewert** J. Dierke ${ }^{\dagger \dagger}$

Institute of Aerodynamics and Flow Technology, Technical Acoustics

German Aerospace Center (DLR)

Lilienthalplatz 7, 38108 Braunschweig, Germany

\begin{abstract}
A lean, swirl-stabilized gas turbine model combustor is simulated with a stochastic approach for combustion noise prediction. The employed hybrid and particle based method, FRPM-CN (Fast Random Particle Method for Combustion Noise Prediction) reconstructs temperature variance based direct combustion noise sources from local CFD-RANS turbulence and flow field statistics. Those monopole sound sources are used as right hand side forcing of the Linearized Euler Equations. First, findings from steady state CFD simulations are validated with experimental results. It is shown that the employed RANS models accurately reproduce the experimental flow field and combustion. Turbulence is treated with a two equation model and a global reaction mechanism is utilized for combustion. Subsequently, the specifications of the CCA (Computational Combustion Acoustics) setup is introduced and selected pressure spectra of the acoustics simulations are compared to experimental results, showing that FRPM-CN is able to deliver absolute combustion noise levels for the investigated burner at low computational costs.
\end{abstract}

\section{Introduction}

Noise emission has become an issue with high social, environmental and economic relevance throughout the last years, especially in the field of aviation. The trend to more quiet aircrafts was already enforced in the early 2000s by for example the organisation ACARE (Advisory Council for Aviation Research and Innovation in Europe) with the formulation of noise emission targets until 2020.1,2 They postulate a reduction of overall levels by $50 \%$ compared to reference values of 2000 . This goal still means a large discrepancy from today's standpoint. However, ACARE goals were taken over in 2011 by a document of the European Comission, called FlightPath 2050. ${ }^{3}$ It extends existing ACARE goals to ambitious $65 \%$ up to 2050, relative to the year 2000. Considering those conditions, from today's status quo, there is a huge interest in fundamental research regarding noise sources and the design of noise reduction measures for aircrafts not only from regulative, political, but also from an industrial point of view.

Figure 1 shows representative aircraft noise components for take-off and approach operation conditions. It becomes clear that the engine noise denotes the most significant contribution to overall noise levels. ${ }^{4,5,6,7}$

\footnotetext{
*Research Associate, DLR, Institute of Combustion Technology, Stuttgart, Germany

†Student, DLR, Institute of Combustion Technology, Stuttgart, Germany

${ }^{\ddagger}$ Research Engineer, DLR, Institute of Combustion Technology, Stuttgart, Germany

$\S$ Research Engineer, DLR, Institute of Combustion Technology, Stuttgart, Germany

IResearch Engineer, DLR, Institute of Combustion Technology, Stuttgart, Germany

$\|$ Professor, DLR, Director of the Institute of Combustion Technology, Stuttgart, Germany

** Research Engineer, DLR, Institute of Aerodynamics and Flow Technology, Technical Acoustics, Braunschweig, Germany

${ }^{\dagger}$ Research Associate, DLR, Institute of Aerodynamics and Flow Technology, Technical Acoustics, Braunschweig, Germany
} 
The engine noise level itself consists of several components. Those are effectively the fan and turbine noise, which have broadband and tonal components as well as the compressor. Furthermore, there are noise emission from the exhaust jet and the combustion chamber, both with mainly broadband character.

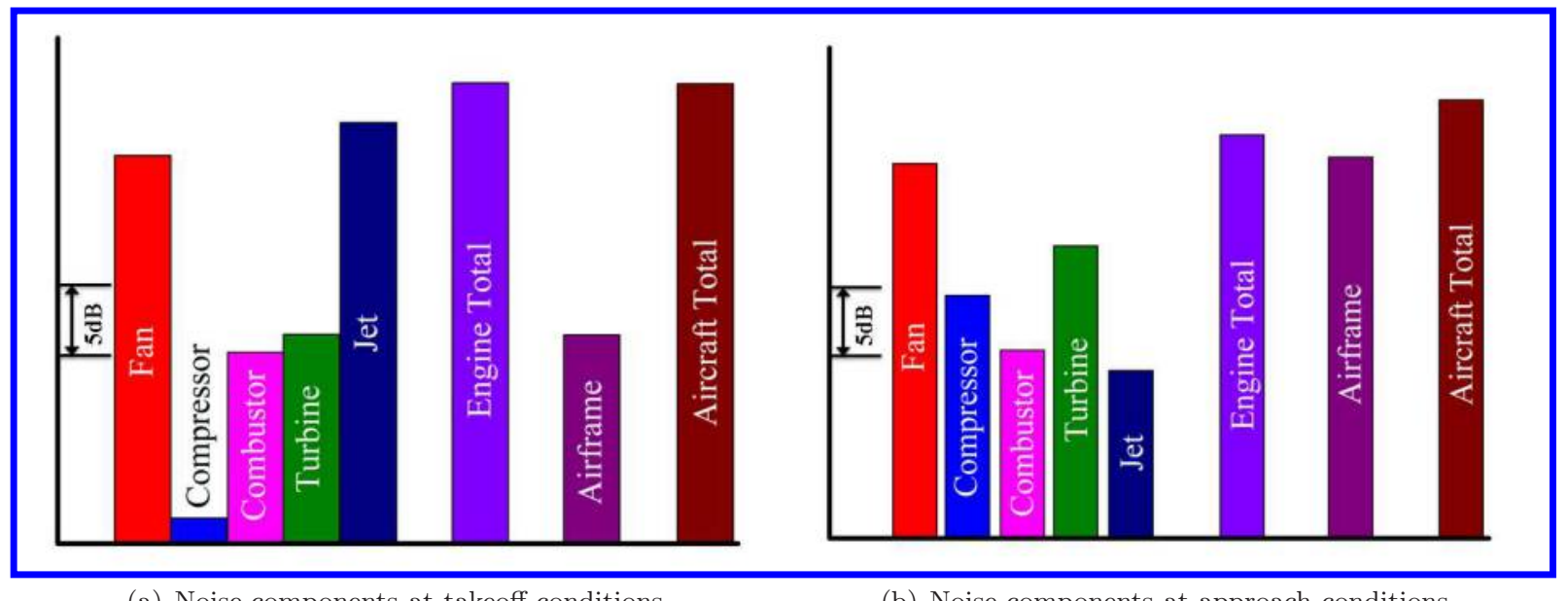

(a) Noise components at takeoff conditions.

(b) Noise components at approach conditions.

Figure 1: Aircraft noise sources at different operation conditions. ${ }^{7,6}$

Overall engine noise levels typically show a more or less broadband distribution, while the single subcomponents contribute at different frequencies: ${ }^{8}$ Jet noise and combustion noise dominate at low frequencies, fan and turbine noise have significant amounts rather in the mid and high frequency regions. Noise levels in Fig. 1 indicate that the contributions of engine subcomponents vary with operation conditions. The biggest differences between take-off and approach for example can be stated for the compressor, turbine and jet noise, while fan and combustion noise contributions stay rather constant in terms of overall levels. However, combustion noise has a significant contribution to overall levels in either case.

From a phenomenological point of view, combustion noise can be subdivided into an indirect and a direct component. Indirect combustion noise is linked to convectively transported entropy spots, while direct combustion noise is linked to heat release flucutations in the reaction zones. The investigations in the presented paper focus on the modeling of direct combustion noise. Its relevance compared to the overall noise levels becomes more clear by looking at recent developments of aircraft engines: ${ }^{9}$ With the introduction of turbofan-engines, a significant reduction of jet noise could be achieved in the past by large bypass flows surrounding the jet. Furthermore, jet noise was reduced by efficiency increase and therefrom resulting lower jet velocities. ${ }^{10}$ In the same turn, the introduction of turbofan engines led to a more significant contribution of fan noise to overall noise levels. On the other hand, in the past few decades, a large amount of research effort was put into the understanding of jet and fan noise and reduction measures as a consequence. This steadily led to an increased importance of combustion noise relative to the overall noise levels. ${ }^{8}$

Further on, latest developments aimed at combustion systems with low pollutant emissions, ${ }^{9,11,12}$ which was achieved by employing premixed or staged combustion. In those novel combustion technologies, the combustion noise phenomenon becomes even more important. In turbulent premixed combustion, there are distinctly higher fluctuations in heat release compared to diffusion flames, especially for operation conditions near the lean blowout limit ${ }^{9,8}$ and therefrom resulting stronger sound emissions. Those technologies are extremely prone to thermoacoustic instability, which in turn dramatically increases pollutant emissions and may lead to severe damaging of the combustion chamber structure. Therefore, the investigation of acoustics of premixed and partially premixed combustion is a research topic with large interest, especially from an industrial point of view.

So it is important to gain a detailed understanding of combustion noise generation mechanisms as a first step in order to be able to derive effective noise reduction techniques. From the numerical or theoretical point of view, the phenomenon of broadband combustion noise due to the interaction of chemical processes with flow unsteadiness can be in principle modeled in two ways, as shown in Fig. 2a: The direct approach, meaning partially or fully scale resolving compressible DNS or LES calculations, which are often difficult to handle and computationally extremely expensive. As an alternative, there are the so called hybrid approaches, separating CFD (Computational Fluid Dynamics) and CCA (Computational Combustion Acoustics ${ }^{13}$ ) scales. 


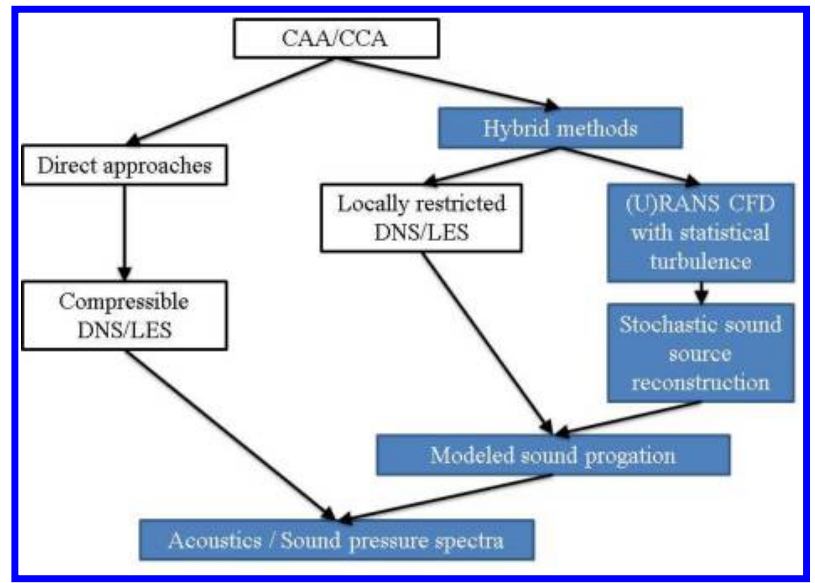

(a) Computational Combustion Acoustics (CCA) numerical approaches.

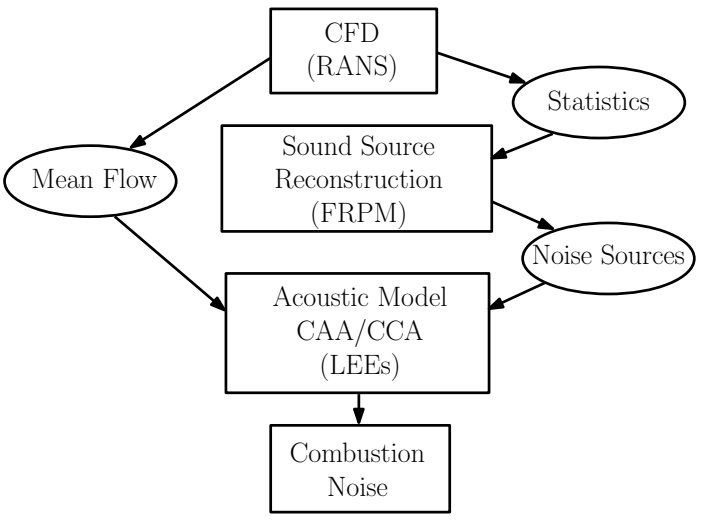

(b) In-principle scheme of the hybrid method FRPM-CN. ${ }^{14}$

Figure 2: Schematic simplified drawings of Computational Combustion Acoustics methods (left) and the functioning of the hybrid method FRPM-CN (right).

They provide a large potential for computational savings and the possibility to apply specifically optimized methods to each part of the problem, ${ }^{15}$ since acoustic pressure fluctuations are usually in the order of magnitude of the CFD computational error.

The hybrid method applied in this work is a time-domain approach, relying on stochastic sound source reconstruction, while sound propagation is computed with the linearized Euler equations (LEEs). The in principle functioning of the method is sketched in Fig. 2b. Reacting CFD RANS simulations are carried out at first, delivering the mean flow, density and pressure background field for the linearized Euler equations and therefore a realistic depiction of refraction effects and sound propagation. At the same time, the CFD RANS simulations provide source field one-point statistics in an assumed source region from the local turbulence quantities. Noise sources are reconstructed with FRPM, which are in this case temperature variance based and the noise sources are coupled to the LEEs as right hand side forcing. The basic equations together with the sound sources denote the overall acoustic model, and its every-timestep solution gives time-signals of acoustic pressure fluctuations, which are transformed to sound pressure spectra at arbitrary observer positions.

The particular line of development for the stochastic, particle based hybrid ansatz with correlated sources which is pursued here started with the introduction of the RPM (Random Particle Mesh Method) by Ewert and Emunds ${ }^{16}$ while Ewert presented further extensions with applications to slat noise, ${ }^{17,18}$ trailing edge and jet noise. ${ }^{19}$ Their RPM realized sources with spatio-temporal correlations based on local turbulence statistics for the applications mentioned previously.

The approach of combustion noise modeling utilized in this work was derived by Mühlbauer et al. ${ }^{20}$ using the sound source reconstruction algorithm from Ewert, ${ }^{16,17,19,18}$ while the derivation of the source term formulation was inspired by the cold jet noise model of Tam and Auriault. ${ }^{21}$ The physical source term model was derived from first principles, using a fundamental pressure-density relation, leading to the linearized Euler energy equation with a right hand side forcing, ${ }^{22}$ while the complete right hand side source expression of the pressure-density relation was taken from Candel et al. ${ }^{13}$ The resulting formulation modeled with RPM was temperature variance based, while the variance field was determined by solving an additional transport equation according to Gerlinger ${ }^{23}$ in the preceding CFD reacting RANS simulations. In a first approach to combustion noise prediction the RPM in conjunction with the acoustic perturbation equations (APEs) were used by Mühlbauer et al. ${ }^{20}$ The genuine APEs were introduced by Ewert and Schröder. ${ }^{24}$ Later the source term model was reformulated on a more general basis, theoretically applicable to all reacting flow cases. ${ }^{25}$ It was derived for the use in combination with the linearized Euler equations and the approach was called RPM-CN (Random Particle Mesh Method for Combustion Noise). ${ }^{26,25,22,27,14}$ Mean flow field data and mean turbulence statistics for this causal approach were provided by steady-state RANS calculations, in view of potential computational savings compared to LES based methods.

On that basis, the method RPM-CN was advanced by Grimm et al. ${ }^{28}$ by using the existing source term 
formulation but a different, highly efficient source reconstruction algorithm which is more suitable for technically relevant applications, the FRPM (Fast Random Particle Method) from Ewert et al. ${ }^{29}$ This approach, the so called FRPM-CN, was verified in terms of one- and two-point source statistics ${ }^{30,28,31}$ as well as farfield spectra reproduction ability ${ }^{30,31}$ with an analytical framework introduced by Ewert et al. ${ }^{32}$ In the presented paper, the full dimensional model is validated for a laboratory combustor application case.

The paper is structured as follows: First, the theoretical framework is set by introducing the employed fundamental equations, turbulence and combustion model. Subsequently, the source term formulation is briefly explained as well as the stochastic method for sound source reconstruction. The procedure of the hybrid method is sketched and followed by the introduction of the laboratory scale combustor validation case. Numerical CFD and CCA setup are explained and accompanied by a validation of used CFD models with experimental data and evaluation of the performance of FRPM-CN in terms of reproduction ability of pressure spectra for a relatively stable operation point of the burner. Computational turnaround times are compared to partially scale resolving LES simulation of a similar operation point.

\section{Thermo-Fluiddymanics Framework}

First, the theoretical framework is set by introducing the basic equations of a combustion system, together with a description of turbulence and combustion model. The reacting CFD simulations are carried out with the finite volume based DLR inhouse code THETA (Turbulent Heat Release Extension of the TAU Code). ${ }^{33}$ A detailed description of the main THETA specifications can be found in the literature. ${ }^{34,35,36}$ The code was originally designed for the simulation of steady and unsteady turbulent reacting flows with an incompressible solver on unstructured meshes using a dualgrid approach. However, in latest works it was extended by pressure based compressible solver approaches ${ }^{37,38,34,35,36}$ in order to be able to properly capture acoustics and thermoacoustic phenomena as well as compressible flow regimes. Therefore, partially scale resolving simulations with THETA serve as comparison cases for the herein carried out simulations with the hybrid approach FRPM-CN in terms of computational turnaround times.

\section{Governing Equations}

The governing equations for a reacting flow in their compressible form - namely the conservation of mass, momentum, energy and a transport equation for the species mass fractions - can be written as

$$
\begin{gathered}
\frac{\partial \rho}{\partial t}+\nabla \cdot(\rho \boldsymbol{u})=0, \\
\frac{\partial(\rho \boldsymbol{u})}{\partial t}+\nabla \cdot(\rho \boldsymbol{u u})+\nabla p=\nabla \cdot \boldsymbol{\tau}_{\tau}, \\
\frac{\partial(\rho h)}{\partial t}+\nabla \cdot(\rho \boldsymbol{u} h)-\frac{\partial p}{\partial t}-\boldsymbol{u} \cdot \nabla p=\nabla \cdot(\lambda \nabla T)+\boldsymbol{\tau}_{\tau}: \nabla \boldsymbol{u}, \\
\frac{\partial\left(\rho Y_{\alpha}\right)}{\partial t}+\nabla \cdot\left(\rho \boldsymbol{u} Y_{\alpha}\right)=\nabla \cdot\left(\boldsymbol{D} \nabla Y_{\alpha}\right)+S_{\alpha},
\end{gathered}
$$

for $\alpha=1,2, \ldots, N_{s}-1$ species with mass fractions $Y_{\alpha}$ and the chemical source term $S_{\alpha}$ associated with each species. $\boldsymbol{\tau}_{\tau}$ and $\boldsymbol{D}$ are the stress and diffusion tensor, while $\lambda$ stands for the thermal conductivity. The term $\boldsymbol{\tau}_{\tau}: \nabla \boldsymbol{u}$ respresents the rate of work for shape change in the case of a constant volume. The component $\nabla \cdot(\rho \boldsymbol{u} u)$ in Eq. (2) is defined as resulting in a column vector after application of the differential operator to the dyadic product $\rho \boldsymbol{u u}$. The same applies for the handling of the tensor of tensions in Eq. $(2), \nabla \cdot \boldsymbol{\tau}_{\tau}$. For a variable density flow, the stress tensor is defined as $\boldsymbol{\tau}_{\tau}=2 \mu\left[\boldsymbol{S}-\frac{1}{3}(\nabla \cdot \boldsymbol{u}) \boldsymbol{I}\right]$, with $\boldsymbol{S}=\frac{1}{2}\left(\nabla \boldsymbol{u}+(\nabla \boldsymbol{u})^{T}\right)$. Pressure $p$ and density $\rho$ are inter-related by the thermal equation of state and the specific gas constant $R$ is expressed in terms of the component mass fractions $Y_{\alpha}$ and molar masses $M_{\alpha}$

$$
\rho=\frac{p_{r e f}+p}{R T} \quad \text { with } \quad R=\mathcal{R} \sum_{\alpha=1}^{N_{s}} \frac{Y_{\alpha}}{M_{\alpha}} .
$$

Equations (1) - (5) describe compressible reacting flow. However, in the context of this work with the hybrid method FRPM-CN, incompressible CFD simulations are conducted. This implies the simplifications 
of the density not being a function of the pressure, the neglection of hydrodynamic pressure fluctuations on energy and no influence of dissipation on energy. ${ }^{23,35}$ However, the employed incompressible equations in the process chain of FRPM-CN can be found in the literature, ${ }^{39,40}$ as well as the formulation for respective turbulence models. The compressible fomulation is preferred here, since the linearized acoustics transport equations of FRPM-CN originate from the primitive form of the fundamental equations. Furthermore, the enthalpy is defined as

$$
h=\int_{T_{0}}^{T} c_{p} d T+\sum_{\alpha=1}^{N_{s}} \Delta h_{f, \alpha}^{0} Y_{\alpha},
$$

with the heat capacity $c_{p}$ and the standard formation of enthalpy $\Delta h_{f, \alpha}^{0}$ at reference conditions for species $\alpha$.

\section{Turbulence Modeling}

The governing equations of a turbulent, reacting flow regime, as introduced in the previous section, describe a multiple multi-scale problem. A dynamic phenomenon, which incorporates a range of fluctuations over several orders of magnitude, is turbulence. In order to fully resolve turbulence dynamics for technically relevant and complex applications, enormous computational effort has to be deployed, especially due to this immense scale disparity. To keep the simulation costs within limits, the fundamental equations (1) - (4) are averaged and turbulence is partially or fully statistically modeled.

Averaging naturally results in a loss of information. ${ }^{23}$ Therefrom, a closure problem ${ }^{36,38}$ for several of the governing equations arises. The unclosed terms are - for the Favre-averaged fundamental equations - the Reynolds stresses $\bar{\rho} \widetilde{\boldsymbol{u}^{\prime \prime} \boldsymbol{u}^{\prime \prime}}$, enthalpie flux $\bar{\rho} \widetilde{\boldsymbol{u}^{\prime \prime} h^{\prime \prime}}$, and mass component fluxes $\bar{\rho} \widetilde{\boldsymbol{u}^{\prime \prime} Y_{\alpha}^{\prime \prime}}$ as well as the chemical source terms $\bar{S}_{\alpha}$ of the transport equations for species mass fractions. The treatment of the unclosed fluxes can be found in the literature, ${ }^{23,14,36,38}$ whereas the modeling of the mean chemical source term will be introduced in the combustion modeling section.

Exchange of momentum in a turbulent flow takes place in vortices. They are modeled via the so called turbulent viscosity. ${ }^{36}$ The underlying theoretical concept comes from Boussinesq. ${ }^{41}$ The Reynolds stresses are accordingly expressed as ${ }^{11}$

$$
-\bar{\rho} \widetilde{\boldsymbol{u}^{\prime \prime} \boldsymbol{u}^{\prime \prime}}=\mu_{t}\left(\nabla \tilde{\boldsymbol{u}}+(\nabla \tilde{\boldsymbol{u}})^{T}-\frac{2}{3}(\nabla \cdot \tilde{\boldsymbol{u}}) \boldsymbol{I}\right)-\frac{2}{3} \bar{\rho} k .
$$

$\boldsymbol{I}$ denotes the identity matrix and $k=\frac{1}{2} \widetilde{\boldsymbol{u}^{\prime \prime} \boldsymbol{u}^{\prime \prime}}$ is the turbulent kinetic energy. Therefore, first and foremost the turbulent viscosity $\mu_{t}$ has to be evaluated. In the context of this work, the two equation $k-\omega$ SST turbulence model in its formulation from Menter ${ }^{42}$ is employed. This involves the solution of two additional transport equations for the turbulent kinetic energy $k$ and the turbulence frequency $\omega$. Essentially, the model consists of a combination of the standard $k-\epsilon$ model $^{43,44}$ in the free field of the flow and the $k-\omega$ model $^{45}$ in near wall regions. The $k-\omega$ SST ansatz connects the $k-\epsilon$ and $k-\omega$ approaches via blending functions in order to exploit the advantages of both models. With the treatment of near wall regions with the $k-\omega$ model, large values of $\omega$ are produced close to the wall which demands a corresponding fine near wall spatial grid resolution. This is especially relevant for complex applications with wall flow detachment phenomena.

\section{Combustion Modeling}

For the laboratory scale combustor treated herein, methane is burned with air under atmospheric conditions. In the framework of the presented paper, reduced so called global reaction mechanisms are used for the modeling of chemical reactions in order to minimize the computational costs and to realize a robust approach for the handling of chemical kinetics.

In such a complex application case, combustion that extends over a wide range of regimes prevails. Those are chemical processes completely dominated by the rate of mixing between fuel and oxidizer $(\mathrm{Da}>>1)$ as one extreme and entirely reaction kinetics dominated processes $(\mathrm{Da}<<1)$ on the other side. Of course, many of the local thermochemical conditions result in a state between the two. In order to adequately treat partially premixed reaction processes, we use a combined EDM/FRC approach for the evaluation of the mean chemical source term for each species $\alpha$. 
It is a combination of the mixing controlled Eddy Dissipation Model ${ }^{46,47}$ (EDM) and the reaction kinetics controlled Finite Rate Chemistry (FRC) ansatz. The overall local resulting reaction rate is determined by the minimum value of the two submodels. Chemical reactions can be generally expressed $\operatorname{as}^{23,48,38,36}$

$$
\sum_{\alpha=1}^{N_{s}} \nu_{\alpha, r}^{\prime} \mathrm{Sp}_{\alpha} \rightarrow \sum_{\alpha=1}^{N_{s}} \nu_{\alpha, r}^{\prime \prime} \mathrm{Sp}_{\alpha}
$$

$N_{s}$ is the total number of species Sp with index $\alpha$ and $\nu^{\prime}, \nu^{\prime \prime}$ denote the stoichiometric coefficients. In this case, only one way reactions are depicted. The combined EDM/FRC approach evaluates the mean chemical source term in Eq. (4) as

$$
S_{\alpha}=M_{\alpha} \sum_{r=1}^{N_{r}}\left(\nu_{\alpha, r}^{\prime \prime}-\nu_{\alpha, r}^{\prime}\right) \cdot \min \left(R R_{r}^{E D M}, R R_{r}^{F R C}\right) .
$$

The reaction rates of EDM alone is

$$
R R_{r}^{E D M}=A \frac{\rho}{\tau_{T}}\left[\min \left(\min _{\alpha, \nu_{\alpha, r}^{\prime} \neq 0} \frac{Y_{\alpha}}{\nu_{\alpha, r}^{\prime} M_{\alpha}}, B \frac{\sum_{\alpha} Y_{\alpha}}{\sum_{\alpha} \nu_{\alpha, r}^{\prime \prime} M_{\alpha}}\right)\right],
$$

with the empirical constants $A=4$ and $B=0.5$. For the EDM, $\tau_{T}^{-1}=\bar{\omega}$ holds. Standalone EDM tends to overpredict reaction rates due to the assumption of infinitely fast chemical reactions, ${ }^{49,38}$ especially in cases with nonequilibrium effects. Therefore it is combined with the Arrhenius based formulation of the FRC source term

$$
R R_{r}^{F R C}=k_{f, r} \prod_{j=1}^{N_{s}} C_{j}^{\nu_{j, r}^{\prime}}-k_{b, r} \prod_{j=1}^{N_{s}} C_{j}^{\nu_{j, r}^{\prime \prime}},
$$

with the concentrations $C_{j}{ }^{35} k_{f, r}$ and $k_{b, r}$ are the forward and backward rate constants which are fitted to the prevailing thermodynamic conditions. As mentioned previously, they are modeled with an Arrhenius expression, assuming the following temperature dependency:

$$
k_{\zeta, r}=A_{r} T^{\beta_{r}} \exp \left(-\frac{E_{a, r}}{\mathcal{R} T}\right), \quad \text { for } \quad \zeta \in[f, b],
$$
$\beta_{r}$.

where $E_{a, r}$ is the activation energy of reaction $r$ with a respective dimensionless temperature exponent

\section{Modeling of Turbulent Combustion Noise}

The main objective of this work is to apply a combustion noise monopole source term based model, which was formulated by Mühlbauer et al. ${ }^{25}$ to a combustor application case. The basic underlying theory as well as the principle functioning of the sound source reconstruction algorithm, FRPM, which was combined with the combustion noise formulation by Grimm et al., ${ }^{30,28}$ is described in the following section.

\section{Source Term Formulation and Acoustic Model}

The basis for the source term formulation derivation is a pressure-density relation

$$
\frac{1}{c^{2}} \frac{D p}{D t}=\frac{D \rho}{D t}+\rho \Phi
$$

with a right hand side expression $\Phi$ from Candel et al. ${ }^{13}$ reading

$$
\begin{aligned}
\Phi= & \frac{\dot{Q}}{\rho c_{p} T}+M \frac{D}{D t}\left(\frac{1}{M}\right)+ \\
& \frac{1}{\rho c_{p} T}\left[\nabla \cdot \lambda \nabla T+\boldsymbol{\tau}_{\boldsymbol{\tau}}: \nabla \mathbf{u}-\sum_{\alpha=1}^{N_{s}} \rho Y_{\alpha} c_{p, \alpha} \mathbf{V}_{\alpha}^{D} \cdot \nabla T\right]
\end{aligned}
$$


with $\lambda$, the heat conductivity and $\mathbf{V}_{\alpha}^{D}$, the diffusion velocity of species $\alpha . \dot{Q}$ is the volumetric heat release rate and $M$ denotes the molecular weight. Eq. (14) is reformulated by making use of the energy equation, leading to a formulation with the heat release rate expressed in terms of temperature change

$$
\Phi=\frac{1}{T} \frac{D T}{D t}-\frac{1}{\rho c_{p} T} \frac{D p}{D t}+M \frac{D}{D t}\left(\frac{1}{M}\right) .
$$

According to Mühlbauer et al., ${ }^{25}$ this formulation is a complete representation of the source term as given by Eq. (14). Since usually applications with low Mach number flows are treated, the first term in Eq. (15) is assumed to be dominant over the second, representing the effects of turbulent velocity fluctuations and the second term is therefore neglected. The third term in Eq. (15) comes into picture only if the average molecular weight of combustion products significantly differs from the educts and is therefore also not considered here for the application of methane-air combustion systems.

Based on the first term of Eq. (15), a right hand side processing rule for the source term expression is obtained by transferring a pressure equation formulation

$$
\frac{D p}{D t}+\gamma p \nabla \cdot \mathbf{u}=\gamma p \Phi
$$

with the isentropic exponent $\gamma=c_{p} / c_{v}$, to the pressure equation form of the linearized Euler equations. ${ }^{22}$ This procedure gives $q_{p}=(\gamma p \Phi)^{\prime}=\rho c^{2} \Phi-\overline{\rho c^{2} \Phi}$, which is then applied to the simplification of Eq. (15). The resulting, temperature variance based source term expression, which is subject to FRPM-CN modeling, reads

$$
q_{p}=\frac{\gamma \bar{p}}{\tilde{T}} \frac{\tilde{D} T^{\prime \prime}}{D t}
$$

For the application of the full scale laboratory combustor simulation, it is coupled with a modified form of the linearized Euler equations

$$
\begin{aligned}
& \frac{\partial \rho^{\prime}}{\partial t}+\widetilde{\boldsymbol{u}} \cdot \nabla \rho^{\prime}+\bar{\rho} \nabla \cdot \boldsymbol{u}^{\prime}=0 \\
& \frac{\partial \boldsymbol{u}^{\prime}}{\partial t}+(\widetilde{\boldsymbol{u}} \cdot \nabla) \boldsymbol{u}^{\prime}+\frac{\nabla p^{\prime}}{\bar{\rho}}=\mathbf{0} \\
& \frac{\partial p^{\prime}}{\partial t}+\widetilde{\boldsymbol{u}} \cdot \nabla p^{\prime}+\gamma \bar{p} \nabla \cdot \boldsymbol{u}^{\prime}=q_{p},
\end{aligned}
$$

with the source term (Eq. (17)) on the right hand side of the pressure equation (Eq. (20)). Eqs. (18) to (20) represent the linearized Euler equations, while meanflow gradient terms are not taken into consideration. As stated by Ewert et al. ${ }^{50}$ those components are assigned to causing instabilities in the LEEs. As observed for the herein treated application case, this is mainly dependent on the local characteristics of the background flow field.

Since the employed combustion noise source term formulation is temperature variance based, an additional transport equation for the temperature variance is solved on top of the numerical CFD RANS solution. It reads

$$
\begin{gathered}
\bar{\rho} \frac{\partial \widetilde{T^{\prime \prime 2}}}{\partial t}+\bar{\rho} \nabla \cdot\left(\widetilde{T^{\prime \prime 2}} \boldsymbol{u}\right)-\nabla \cdot\left[\left(\frac{\mu}{P r}+\frac{\mu_{t}}{P r_{t}}\right) \nabla \widetilde{T^{\prime \prime 2}}\right] \\
=\underbrace{2 \frac{\mu_{t}}{P r_{t}}(\nabla \widetilde{T})^{2}}_{\text {production }} \underbrace{-\bar{\rho} C_{T} \frac{\widetilde{T^{\prime \prime 2}}}{\tau_{T}}}_{\text {dissipation }},
\end{gathered}
$$

with the model constant $C_{T}=2 . \mu$ and $\operatorname{Pr}$ are the viscosity and the Prandtl number. The indices $t$ and $T$ mean turbulent and turbulent temperature associated. The $\sim$ denotes Favré averaging. 


\section{Stochastic Reconstruction of Sound Sources}

The source term in Eq. (17) is subject to stochastic sound source reconstruction. Therefore, the Fast Random Particle Method for Combustion Noise, introduced by Grimm et al. ${ }^{30,28}$ is employed. Sound sources are built for each timestep according to local turbulence statistics from convective white noise. This noise field is realized in a discrete form by mapping random values carried by floating particles onto a source field grid. The spatial extensions of the source field are chosen according to the characteristics of the solution of Eq. (21). The spatial filtering algorithm of sources can be generally described by

$$
Q(\boldsymbol{x}, t)=\int_{V_{s}} \hat{A}(\boldsymbol{x}) \mathcal{G}\left(\left|\boldsymbol{x}-\boldsymbol{x}^{\prime}\right|, l_{T}(\boldsymbol{x})\right) \mathcal{U}\left(\boldsymbol{x}^{\prime}, t\right) d^{3} \boldsymbol{x}^{\prime} .
$$

The discretization with FRPM instead of a preceding, streamline based approach RPM, ${ }^{22}$ features an initialization with particles in the whole source region and therefore provides the resolution of recirculation zones. Furthermore, the FRPM grid is orthogonal in contrary to the streamline-based RPM discretization and therefore highly efficient Purser filter ${ }^{51,52}$ are employed for the source filtering, represented by $\mathcal{G}$, which is convoluted with a white noise field $\mathcal{U}$. Integration of source components is performed over the source volume $V_{s}$ and the local amplitude scaling is realized according to $\hat{A}=\sqrt{\hat{R}(\boldsymbol{x}) / l_{T}^{3}(\boldsymbol{x})}$, in order to achieve the appropriate local source variance. The spatial white noise field $\mathcal{U}$ is processed with a first order Langevin approach in time for the realization of turbulence induced decay,

$$
\frac{D_{0}}{D t} \mathcal{U}=-\frac{1}{\tau_{T}} \mathcal{U}+\sqrt{\frac{2}{\tau_{T}}} \xi(\boldsymbol{x}, t) .
$$

Equation (23) is a stochastic differential equation, realizing a long-term drift behaviour with the first component on the right hand side, while the second - so called diffusion term - introduces a Gaussian distributed white noise forcing, for which the random values have to be chosen appropriately. ${ }^{19} D_{0} / D t=$ $\partial / \partial t+\boldsymbol{u}_{0}^{c} \cdot \nabla$ and $\boldsymbol{u}_{0}^{c}$ is the mean CFD RANS velocity field. For $\xi(\boldsymbol{x}, t)$, the properties

$$
\begin{aligned}
\langle\xi(\boldsymbol{x}, t)\rangle & =0, \\
\langle\xi(\boldsymbol{x}, t) \xi(\boldsymbol{x}+\boldsymbol{r}, t+\tau)\rangle & =\delta\left(\boldsymbol{r}-\boldsymbol{u}_{0}^{c} \tau\right) \delta(\tau)
\end{aligned}
$$

hold. The brackets \langle\rangle denote ensemble-averaging. For small separation distance $\boldsymbol{r}$ and time $\tau$, the noise field $\mathcal{U}$ correlation can be expressed by taking into account the solution of Eq. (23) and the source statistics from Eq. (25), as

$$
\langle\mathcal{U}(\boldsymbol{x}, t) \mathcal{U}(\boldsymbol{x}+\boldsymbol{r}, t+\tau)\rangle=\delta\left(\boldsymbol{r}-\boldsymbol{u}_{0}^{c} \tau\right) \exp \left(-|\tau| /_{T}\right),
$$

or in words, the Langevin process induces an exponential decorrelation, representing turbulence related decay. The overall FRPM-inherent correlation function, resulting from the Langevin-induced decorrelation and the use of a Gaussian-shaped filter for the sound reconstruction procedure resulting from Eq. (22), reads

$$
\mathcal{R}(\boldsymbol{x}, \boldsymbol{r}, \tau)=\hat{R}(\boldsymbol{x}) \exp \left(-\frac{|\tau|}{\tau_{T}}-\frac{\pi}{4 l_{T}^{2}(\boldsymbol{x})}\left|\boldsymbol{r}-\boldsymbol{u}_{0}^{c} \tau\right|^{2}\right) .
$$

Equation (27) is used as the correlation function of combustion noise sources for the presented numerical simulations with $\hat{R}(\boldsymbol{x})=\widetilde{T^{\prime \prime 2}}(\boldsymbol{x}) / \tau_{T}(\boldsymbol{x})^{2} . \boldsymbol{r}$ and $\tau$ in Eq. (27) are the separation distance and time, respectively, while $\tau_{T}$ and $l_{T}$ are the local turbulent time- and lengthscale, herein taken from the reacting CFD RANS solution.

\section{Laboratory Scale Combustor Application Case}

The numerically investigated PRECCINSTA (Prediction and Control of Combustion Instabilities in Industrial Gas Turbines) burner is schematically shown in Fig. 3a. The numerical simulations are validated based on an experimental reference study with technical and perfect premixing by Dem et al. ${ }^{53}$ However, the validation in the framework of this paper mainly focuses on the technically premixed combustion regime. The burner works as follows: 
An air plenum is mounted upstream of the burner. Air is led into the plenum and consecutively through a swirler into the combustion chamber. The radial swirler consists of 12 swirler vanes. The fuel is induced from an additional fuel plenum into the swirler vanes in a jet-in-crossflow arrangement. ${ }^{53}$ Fuel and oxidizer then partially mix in the range of a converging nozzle, which is characterized by a conical center body. The tip of the center body denotes the reference point of the global coordinate system, as indicated in Fig. 3 b. Subsequently, the air-fuel mixture expands into the combustion chamber, which has the dimensions $85 \mathrm{~mm} \times 85 \mathrm{~mm} \times 114 \mathrm{~mm}$. The burnt gas exits the combustion chamber through a converging nozzle. A detailed description of the experimental setup can be found in the literature. ${ }^{53}$

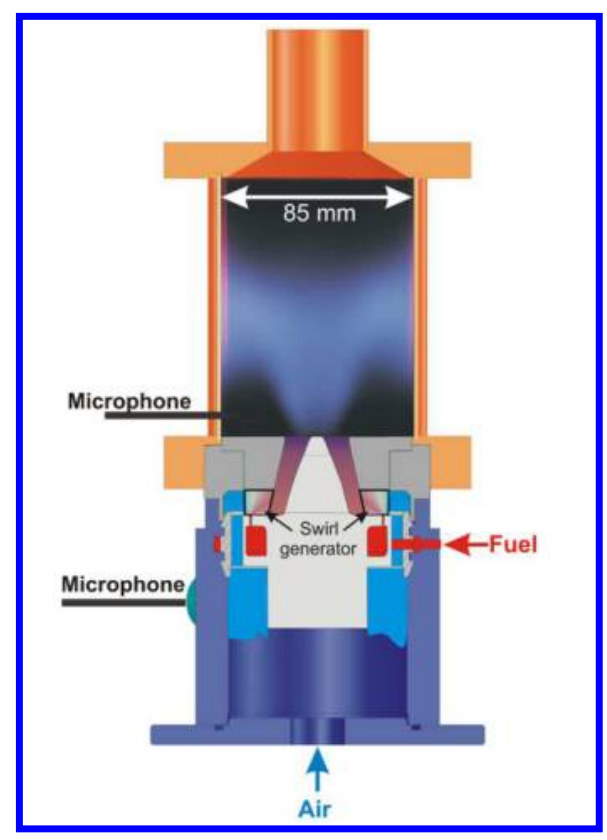

(a) Schematic drawing of the burner. ${ }^{5}$

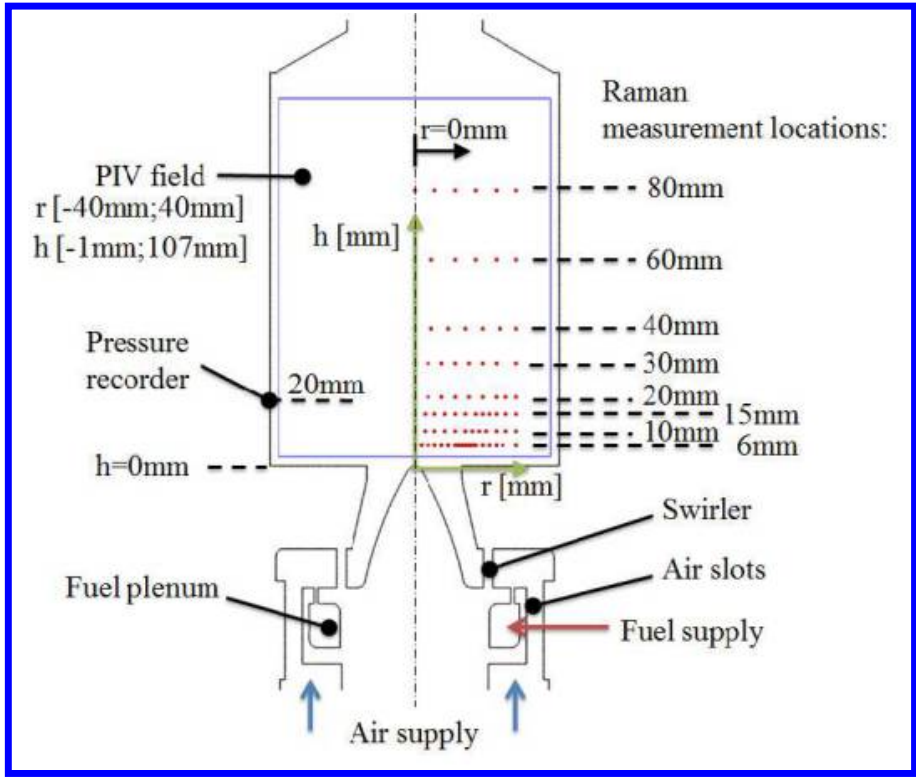

(b) Spatial conventions and measurement locations for Raman and PIV.

Figure 3: Schematic drawing of the investigated PRECCINSTA burner and measurement locations.

Validation is carried out for the flow field with averaged results from stereoscopic particle image velocimetry (PIV) ${ }^{53,54}$ of all three velocity components at horizontal profile lines in the combustion chamber at $h=6 \mathrm{~mm}, 10 \mathrm{~mm}, 20 \mathrm{~mm}, 40 \mathrm{~mm}$. Single-shot laser Raman scattering measurements ${ }^{55,56}$ are available for temperature profile lines and the mean species concentrations $\left(\mathrm{O}_{2}, \mathrm{CH}_{4}, \mathrm{CO}_{2}, \mathrm{H}_{2} \mathrm{O}\right)$ at the same positions for validation of combustion and reaction mechanism. Mean $\mathrm{OH}$ chemiluminescence and acoustic pressure measurements are available for a wide range of operation conditions. Acoustic fluctuating pressure is sampled at a combustion chamber reference microphone position at $x=42.5 \mathrm{~mm}, y=r=42.5 \mathrm{~mm}, z=h=20 \mathrm{~mm}$.

Since the numerical validation studies focus on the broadband combustion noise phenomenon, the selected operation condition is based on an acoustically relatively stable case. Fig. 4 shows a wide range of operation points for partially premixed combustion of the PRECCINSTA burner, ranked by thermal power and equivalence ratio.

As indicated in Fig. 4a, the burner produces different mean flame shapes, as comes out of OH chemiluminescence measurements. For higher equivalence ratios, a V-shape of the flame can be observed, regardless of the degree of thermal power. From our experience, the V-shaped flame is usually anchored at the tip of the conical bluff body and is present at thermoacoustically stable operation points. At lower equivalence ratios and therefore leaner combustion, the flame exhibits a characteristic M-shape and those operation points are considered more unstable. Those operation conditions also usually show a distinct flow instability, emerging in the swirler region, the so called PVC (Precessing Vortex Core), as shown by Oberleithner et al. ${ }^{54}$ This unsteady phenomenon cannot be depicted in steady state CFD simulations and causes additional transient flowfield-flame interaction effects in the system. Furthermore, the lifted M-shape flames are difficult to reproduce in steady state CFD RANS simulations, since the mean flame shape in unstable cases results from 


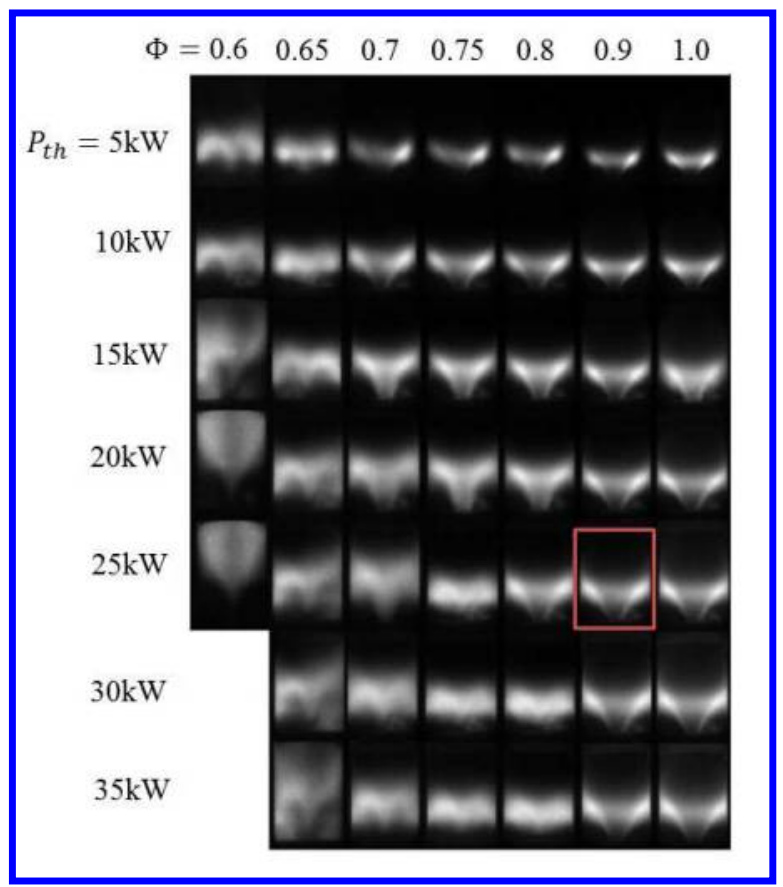

(a) Flame shape.

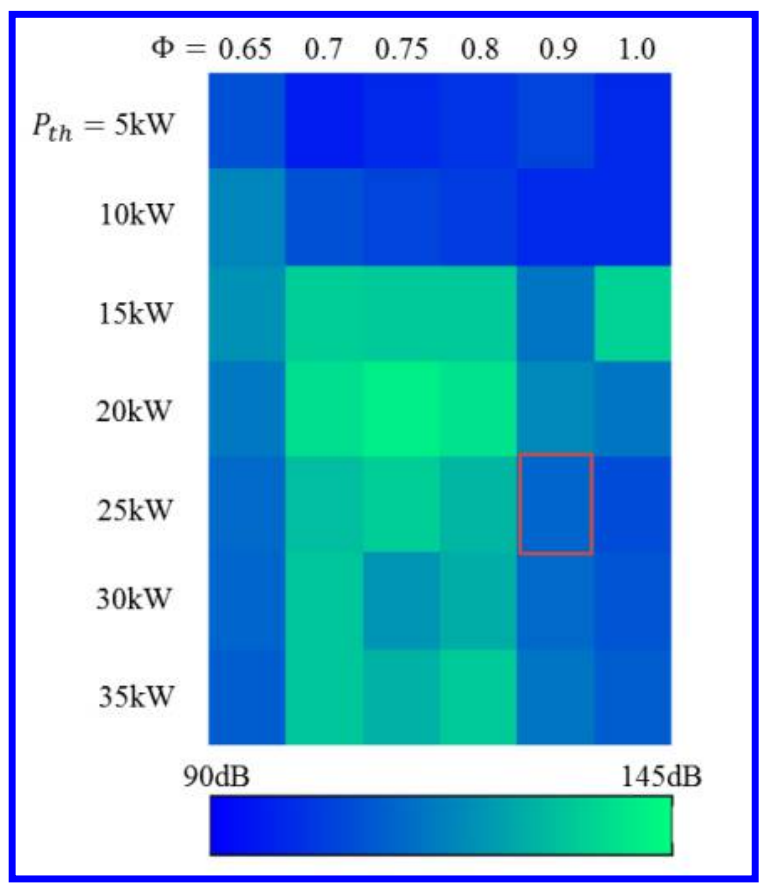

(b) Maximum levels of acoustic pressure amplitudes.

Figure 4: Flame shape and characteristic acoustic pressure amplitudes for different thermal powers $P_{t h}$ and equivalence ratios $\Phi .{ }^{57,56}$

large axial flow field and flame oscillations due to strong Helmholtz eigenmodes.

Fig. $4 \mathrm{~b}$ shows a stability map of the burner in the respective operation ranges corresponding to Fig. 4a. It estimates acoustic stability of the burner by monitoring the largest present sound pressure level in $\mathrm{dB}$. It confirmes the previous statement that the burner operates in relatively stable conditions for larger equivalence ratios but unstable operation for leaner combustion regimes. From the point of view of investigating direct combustion noise only, a preferably stable operation point at $P_{t h}=25 \mathrm{~kW}$ and $\Phi=0.9$ with a global mixture fraction of $f=0.0498$ is chosen, which also exhibits a V-shaped flame. Air is induced through the upstream air inlet at a mass flow rate of $\dot{m}_{a i r}=574 \frac{\mathrm{g}}{\mathrm{min}}$, while the fuel is induced from the fuel plenum with $\dot{m}_{\text {fuel }}=30 \frac{\mathrm{g}}{\mathrm{min}}$ at atmospheric conditions.

\section{A. Numerical CFD Setup}

The unstructured grid for the reacting CFD RANS simulations ${ }^{58}$ is shown in Fig. 5. The regions of the reaction zone as well as the swirler vanes and the connecting ribs between air plenum and combustor are refined compared to the air plenum and downstream zones in the combustion chamber and the exhaust tube. The inner computational domain is entirely discretized with tetrahedra. Near wall regions in the swirler and combustion chamber are covered with three prism layers and they are interconnected to the inner domains via pyramid elements. The thetrahedral grid consists of $16.6 \mathrm{M}$ elements with $2.9 \mathrm{M}$ points. Furthermore, it consists of $0.47 \mathrm{M}$ prism elements and $5 \mathrm{~K}$ pyramids. The grid is most refined in regions where fuel is injected in the swirler vanes via thin tubes from the fuel plenum.

The simulations are conducted with the finite volume based DLR inhouse code THETA. ${ }^{59,34}$ The reacting CFD RANS calculations are based on an incompressible formulation of the basic Eqs. (1) to (4), treated with a SIMPLE (Semi-Implicit Method for Pressure Linked Equations) solution procedure. For the spatial discretization of the steady state RANS simulations, a first order accurate upwind scheme (UDS) is used. In case an URANS is performed, second order accurate upwind (QUDS) in space and a three point backward scheme (TPB) in time is chosen. The physical timestep in intermediate URANS simulations is $\Delta t=1 \cdot 10^{-5} \mathrm{~s}$. As introduced previously, the $k \omega$-SST turbulence model ${ }^{42}$ is employed.

Mass flow is specified at air and fuel inlets with a turbulence degree of $T u=0.01$ and an initial turbulent lengthscale $l_{T}=1 \cdot 10^{-4} \mathrm{~m}$, respectively, at $T_{i n}=320 \mathrm{~K}$. At the combustion chamber outlet tube, the 


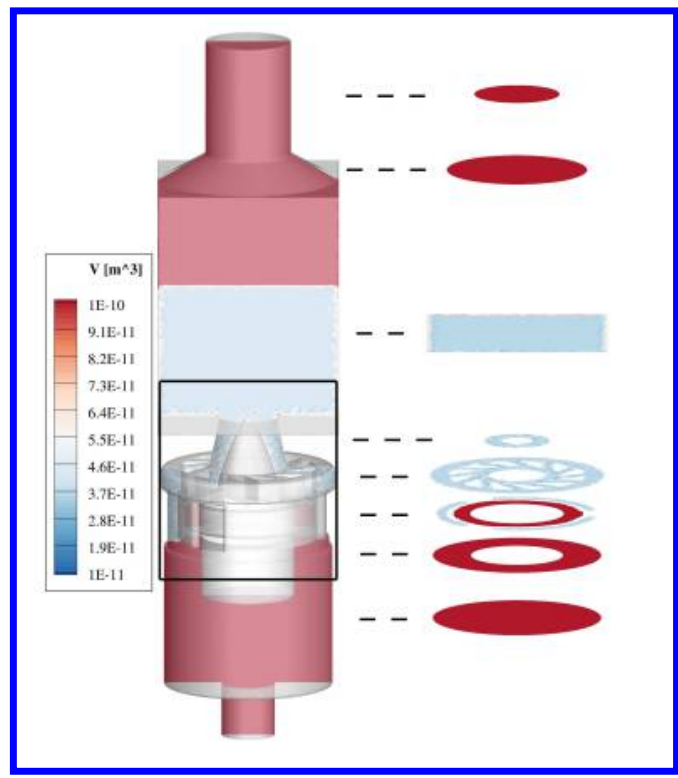

(a) Mid-plane and horizontal intersecting planes of the computational CFD domain with local cell volume. Indication of detailed view in Fig. 5b.

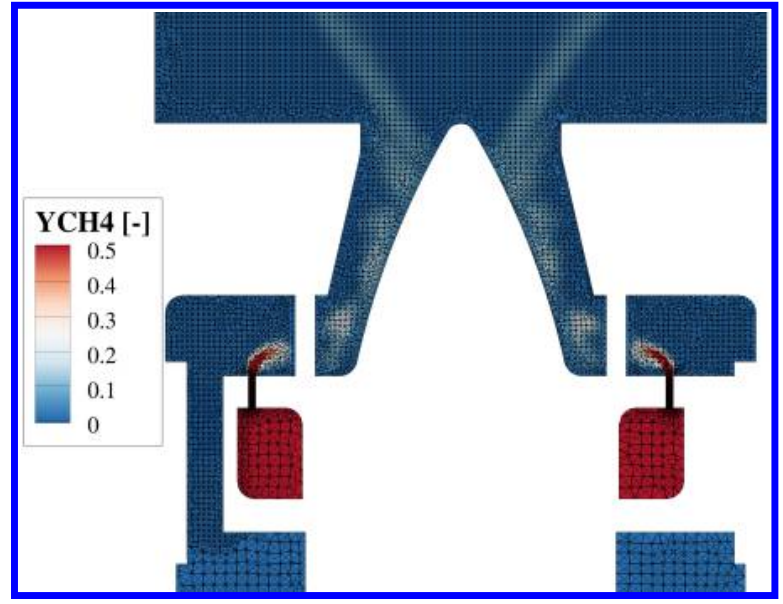

(b) Detailed view. Spatial resolution of the swirler and combustion chamber with prism layers and underlying mass fraction of $\mathrm{CH}_{4}$.

Figure 5: Computational CFD domain. ${ }^{58}$

static pressure is set to ambient reference conditions. Walls are treated as adiabatic except for isothermal combustion chamber walls, impinged with $T_{W}=1500 \mathrm{~K}$.

Monitoring positions of the mean flow field and combustion quantities are horizontal profile lines according to the respective PIV measurements at $h=0.006 \mathrm{~m}, 0.01 \mathrm{~m}, 0.02 \mathrm{~m}, 0.04 \mathrm{~m}$. As a solution strategy, at first, steady state reacting simulations are carried out. In order to accurately predict chemical reactions, an intermediate URANS simulation with a physical timestep is attached in order to get a reasonable temperature field. This is in turn followed by another steady state RANS simulation, the flow and combustion statistics of which are taken for the later CCA sound source reconstruction.

Combustion is treated with a combined EDM/FRC approach for global reaction schemes. In this work, methane is burned with air under atmospheric conditions. Therefore a single-step global reaction mechanism proposed by Westbrook and Dryer ${ }^{60}$ is employed. On top of the steady state solution of flow field and combustion, an additional transport equation, Eq. (21), for the determination of a field solution for the temperature variance is solved. Therefrom, the local source term fluctuation amplitude in the subsequent CCA simulations is determined.

\section{B. CFD Results}

First, a general validation study concerning flow field and combustion is carried out. Fig. 6 shows rhmidplane results for absolute (left) and axial (right) velocity. The overlay of CFD contours and PIV isolines of velocity in Fig. 6a reveals that the RANS gives an excellent qualitative reproduction of the experimental velocity field. Spatial patterns especially in the inner recirculation zone nicely match and the opening angle of the flow after entering the combustion chamber is almost exactly reproduced in the numerical simulation.

A similar quality of agreement between simulation and experiment can be seen in Fig. 6b. It shows axial velocity contours from the simulation with zero-lines of velocity and therefore an indication for the extension of recirculation zones. Present deviations are mainly far downstream in the combustor, while the regions close to the expansion of the flow are accurately captured. The same quality of reproduction can be stated for the outer recirculation zones, as far as data is available from the experiment.

A quantitative comparison of CFD and experiment is shown in Fig. 7. The numerical simulation gives a fairly good representation of the experimentally evaluated flow field for all velocity components. However, two deviations to experiments can be stated for almost all the profiles. Especially close to the combustor inlet, CFD profiles underpredict experimental values, most probably due to the low order of spatial discretization 


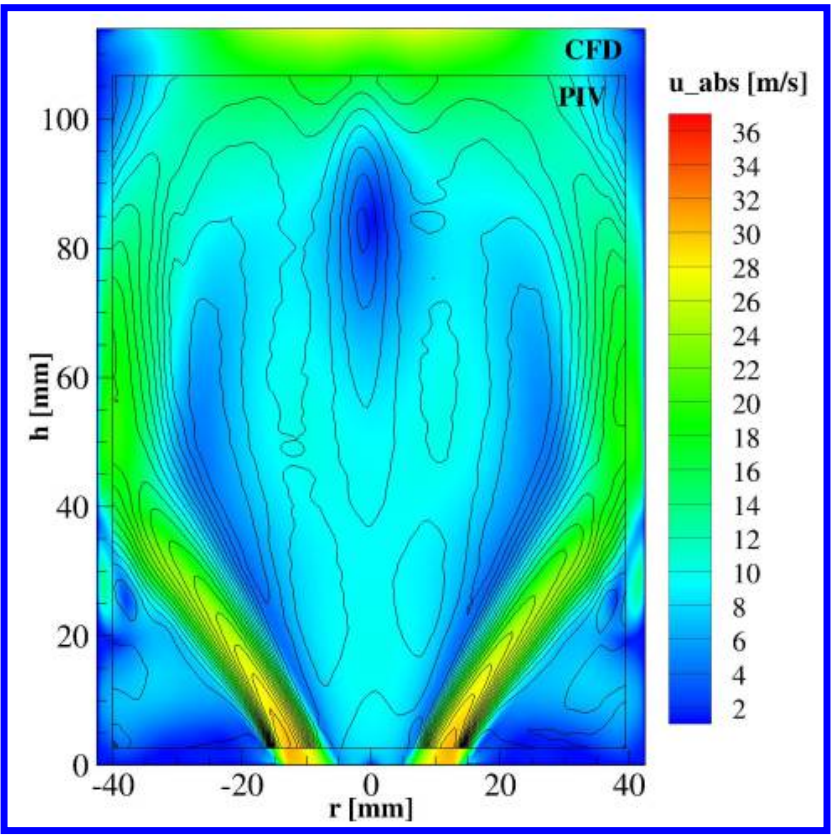

(a) Combustor midplane, absolute velocity. Contours: CFD solution, Isolines: PIV measurements.

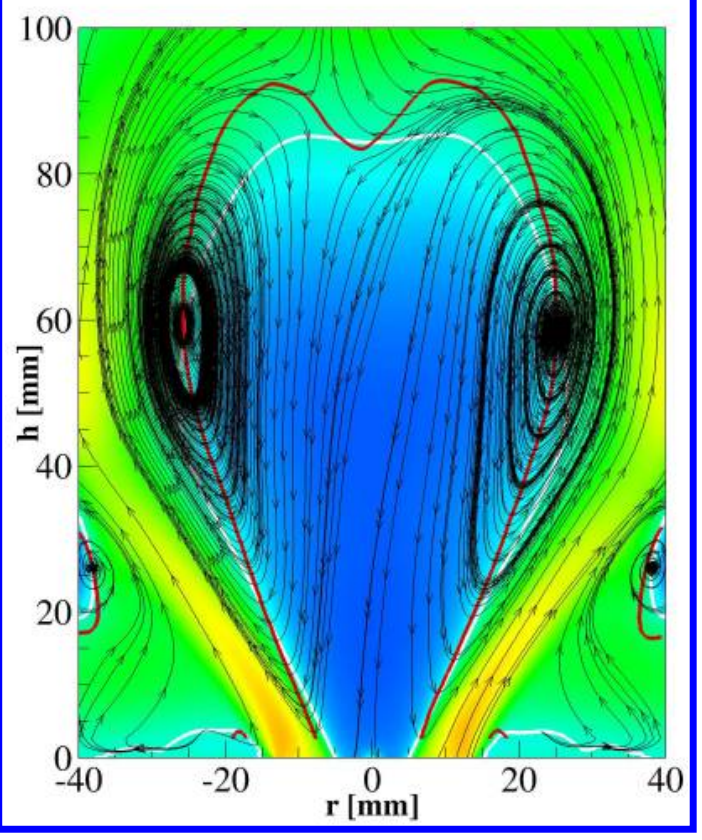

(b) Combustor midplane, axial $x$-velocity component. Indication of recirculation zones. White: CFD RANS, Red: PIV. Streamlines from averaged PIV data.

\section{Figure 6: Qualitative comparison of flow field of CFD simulation and PIV measurements.}

in the simulation and the thereby induced numerical diffusion.

Furthermore, the simulation overpredicts the opening angle of the flow, especially downstream of the reaction zone. Peak positions of velocity are almost exactly reproduced close to the expansion of the flow into the combustion zone. Since the source volume of combustion noise sources is located close to the combustion chamber inlet, the CFD solution gives an accurate flow field representation for the convection of sources and the depiction of background flow field for a physical propagation of sound waves. From our point of experience, the underprediction of peak values of velocity components in the simulation does not significantly affect the resulting pressure spectra of the computational combustion acoustics simulations.

A comparison of CFD temperature and RMS of temperature with Raman measurements is shown in Fig. 8. Raman measurement locations are indicated with black dots, while the intermediate field values are interpolated with a statistical Kriging algorithm. ${ }^{61}$ As can be seen in Fig. 8a, the flame is located in the inner recirculation zone and anchored at the tip of the bluff body in the conical nozzle prior to the combustion chamber. Accoring to the quality of flow field reproduction, the V-shape of the flame is nicely captured. However, there are certain deviations in peak values of temperature, which are assumed to be linked to the employment of a simplified global reaction mechanism. Similar characteristics can be observed from the comparison of RMS temperature values in Fig. 8b. The RMS profiles of the experiment are qualitatively nicely matched, but the peak values are overpredicted in the simulation.

The quantitative comparison of temperature and RMS of temperature in Fig. 9 confirms the trends observed in Fig. 8. The flame shape especially on profile lines close to the flame root is nicely captured, while it opens up slightly too far for downstream profiles. Reaction rates appear to be slightly overpredicted by the combined EDM/FRC approach, since the flame sits too far upstream and therefore temperature peak values especially near the burner axis are too high.

As stated previously, temperature variance profiles are evaluated from equation Eq. (21), which determines the field solution from an existing flow and temperature field. Therefore, the temperature RMS values follow the same trends as the temperature and flow field profiles. Variance peak values are accordingly overpredicted due to the overshoot of maximum temperature levels and therefrom resulting larger local temperature gradients. However, the temperature variance profiles show a reasonable agreement with 


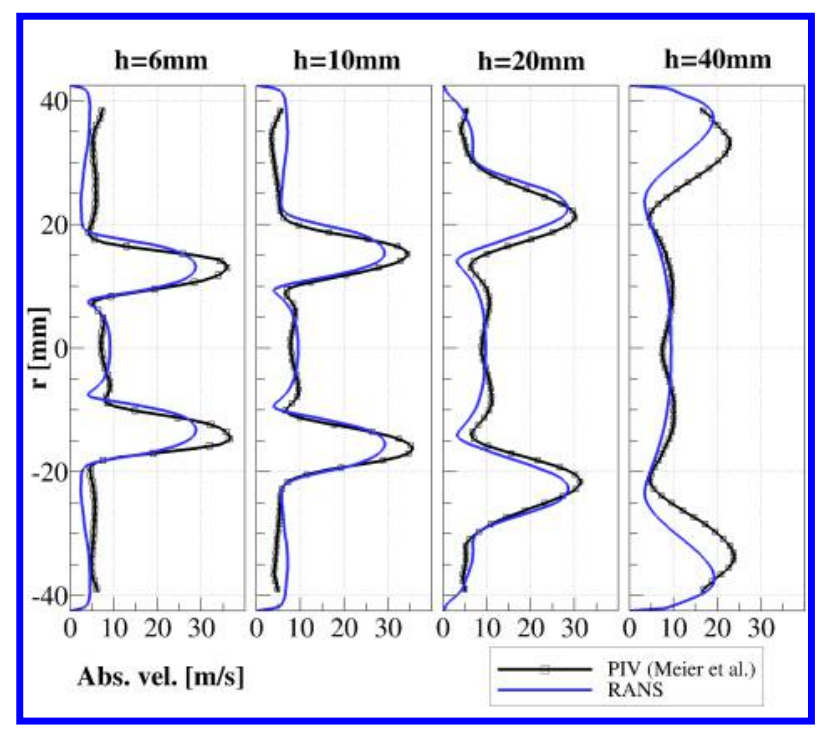

(a) Absolute velocity $|\boldsymbol{u}|\left[\frac{\mathrm{m}}{\mathrm{s}}\right]$.

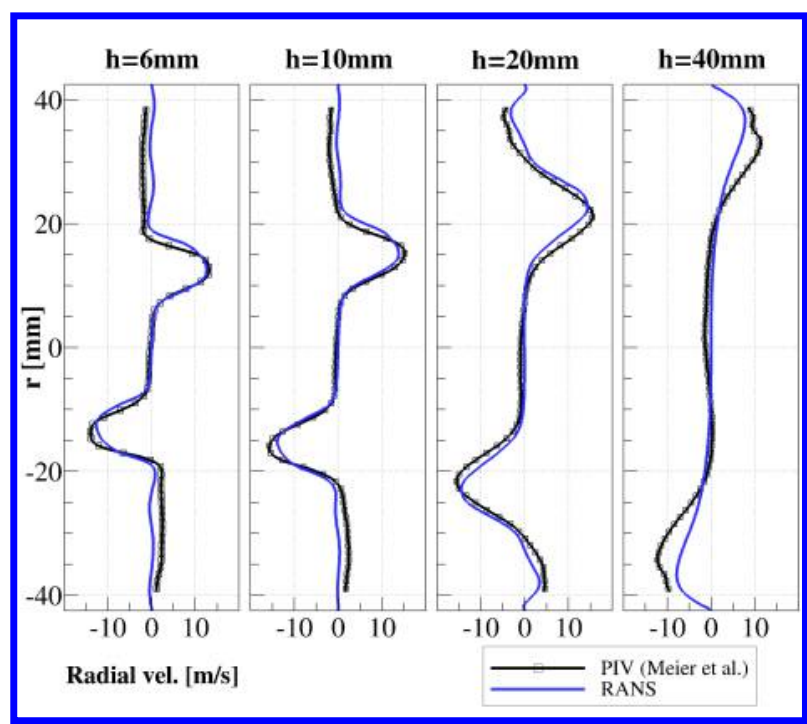

(c) Radial velocity $\left[\frac{\mathrm{m}}{\mathrm{s}}\right]$.

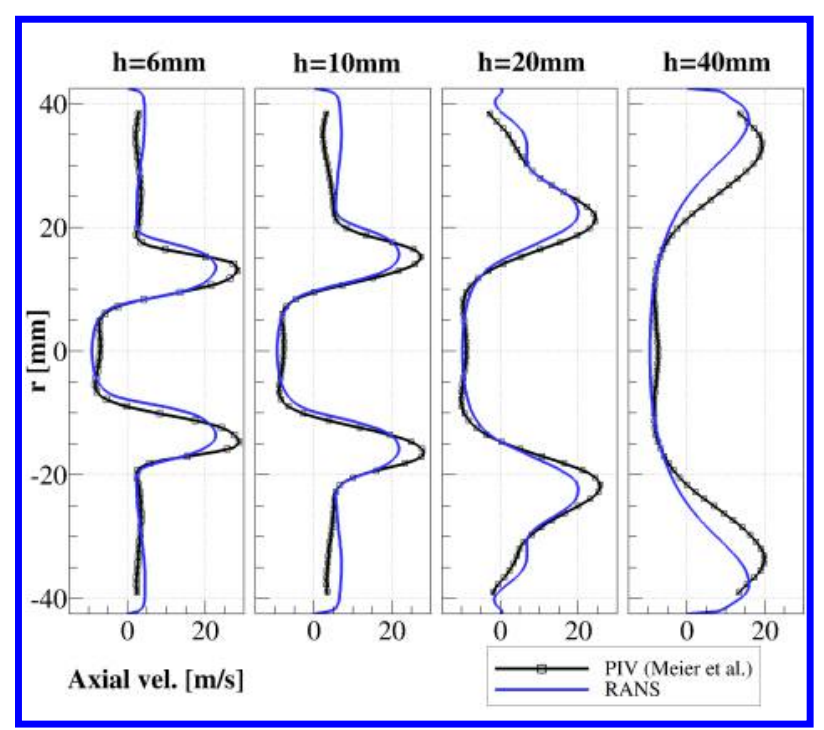

(b) Axial velocity $\left[\frac{\mathrm{m}}{\mathrm{s}}\right]$.

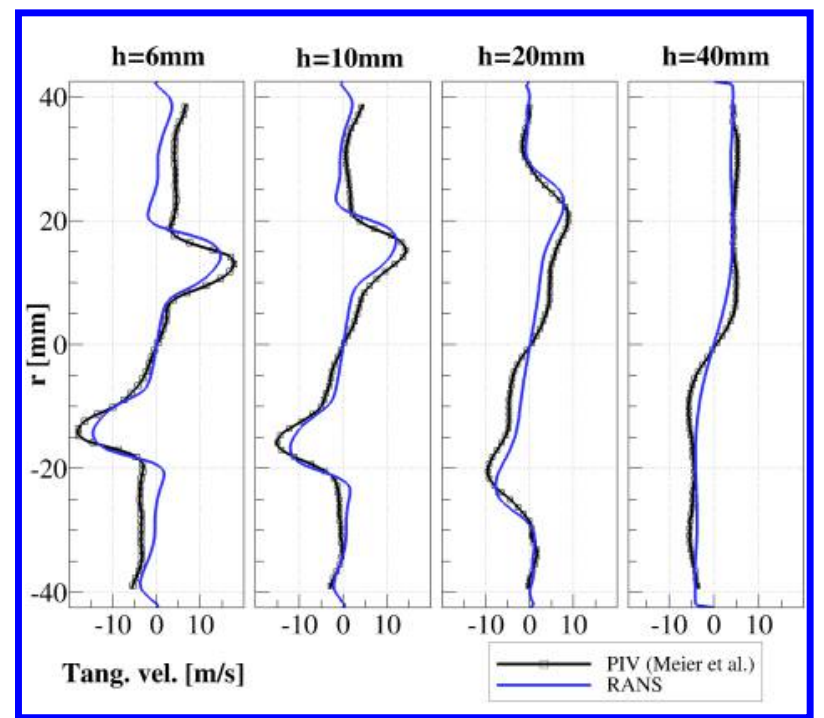

(d) Tangential velocity $\left[\frac{\mathrm{m}}{\mathrm{s}}\right]$.

Figure 7: Comparison of velocity components of CFD RANS simulation and PIV experiment at horizontal profile lines in the combustion chamber. 


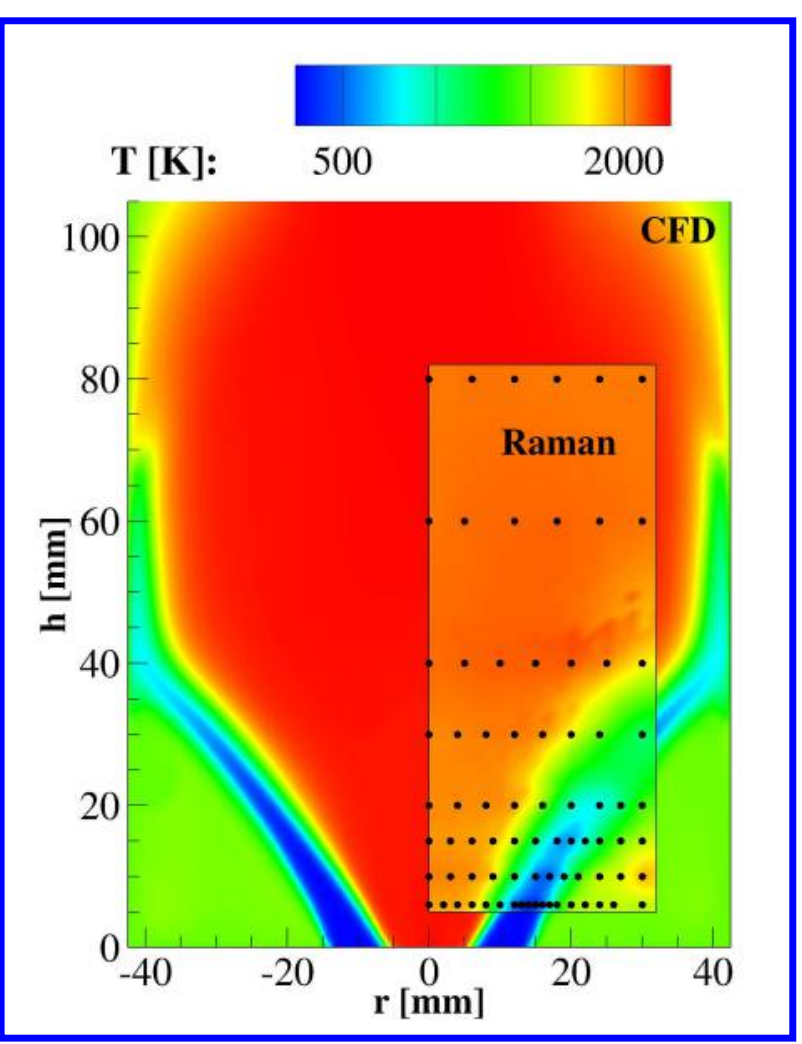

(a) CFD contour plot of temperature and an overlay of Raman measurement results.

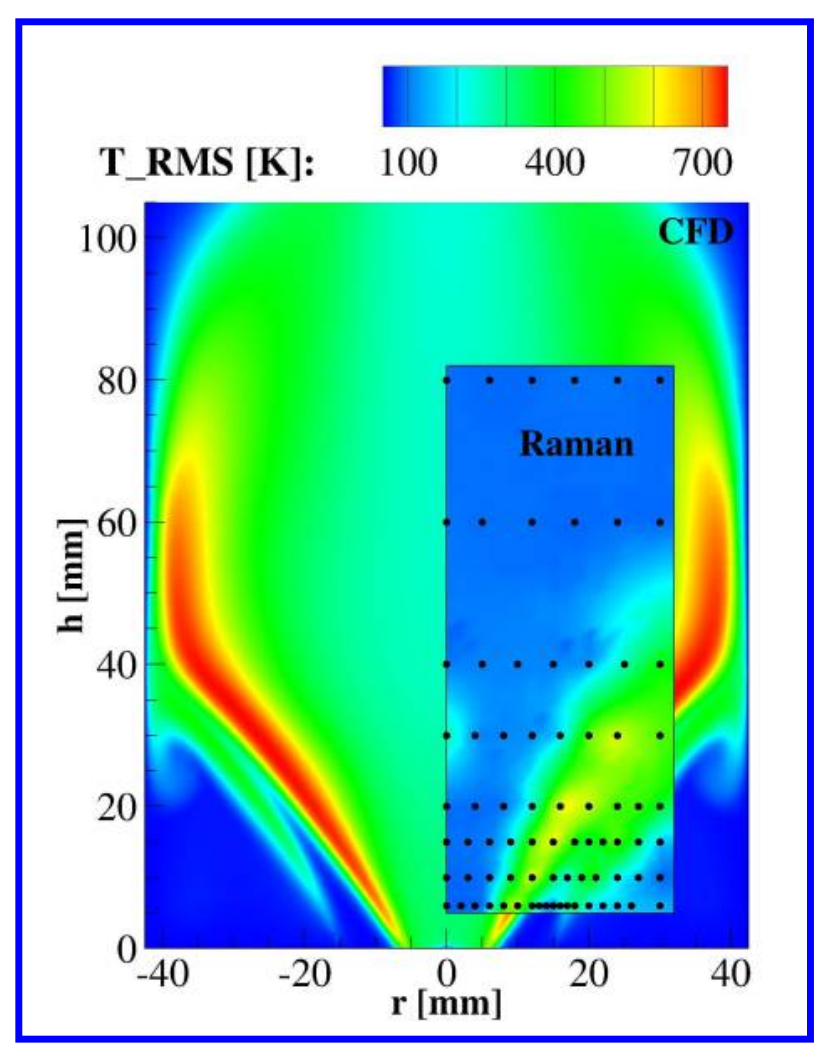

(b) CFD contour plot of RMS of temperature and an overly of Raman measurement results.

Figure 8: Quantitative comparison of combustion of CFD simulation and Raman measurements.

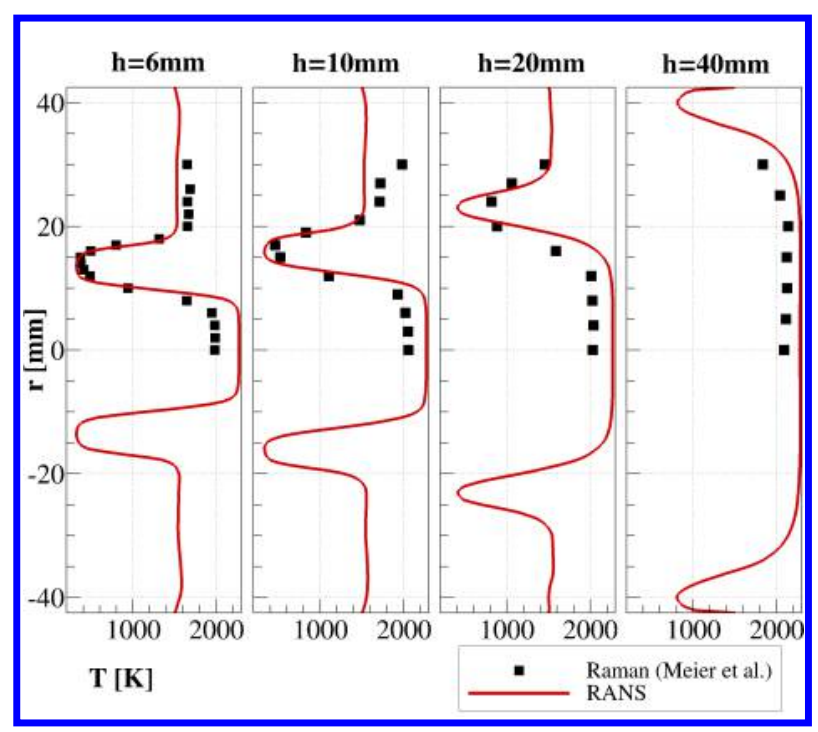

(a) Temperature $[\mathrm{K}]$.

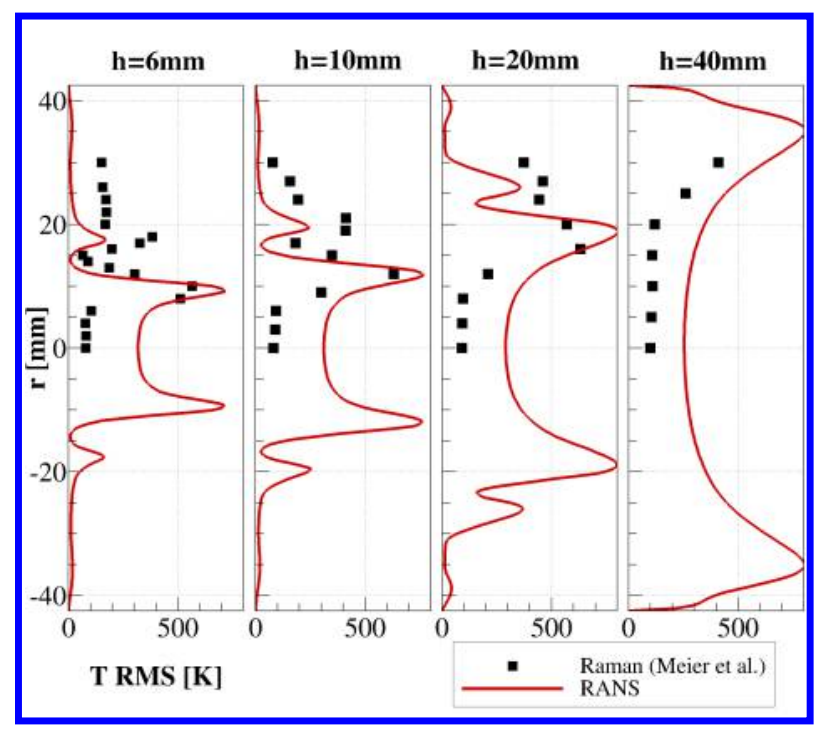

(b) RMS of temperature $[\mathrm{K}]$.

Figure 9: Comparison of temperature and temperature RMS profiles at horizontal profile lines in the combustion chamber. 
the experimental data, considering the low computational cost of a steady state RANS simulation. Therefore, they are employed as a basis for the local amplitude scaling of source fluctuation of the consecutively reconstructed combustion noise sources, formally described by Eq. (17).

\section{Computational Combustion Acoustics Setup}

Computational combustion acoustics simulations are carried out with the DLR inhouse code PIANO (Perturbation Investigation of Aerodynamic Noise). ${ }^{62}$ PIANO is employed for the sound propagation modeling via linearized Euler equations. Sound sources are reconstructed from local turbulence statistics as an input from the preceding CFD RANS simulations. First, the numerical setup for sound propagation modeling is introduced, followed by the configuration for sound source reconstruction of combustion noise sources with the integrated module FRPM (Fast Random Particle Method).

The computational grid for the acoustics simulations is shown in Fig. 10. The finite-difference based dispersion relation preserving Scheme (DRP) from Tam \& $\mathrm{Webb}^{63}$ is employed for spatial discretization. Therefore, the block-structured grid consists of $1.5 \mathrm{M}$ hexahedra with $1.94 \mathrm{M}$ nodes in 938 blocks. It spatially resolves frequencies up to $f_{\max }=15 \mathrm{kHz}$. For solution proceeding in time, a classical four-step Runge Kutta scheme is used. The mesh is optimized with respect to the local growth rate of adjacent cells with a maximum growth rate in critical regions of 1.05 . Those are zones with large velocity and density gradients close to the combustor inlet or the swirler vanes, where fuel is added to air in a jet-in-crossflow alignment. Mean flow field quantities $\bar{\rho}, \tilde{u}, \tilde{v}, \tilde{w}, \bar{p}$ are interpolated from the unstructured CFD RANS grid to the CCA domains using a statistical Kriging ${ }^{61}$ algorithm. The mean flow density field in the swirler vanes is smoothed for stability reasons, to avoid large density gradients in adjacent cells due to the thin fuel jet.

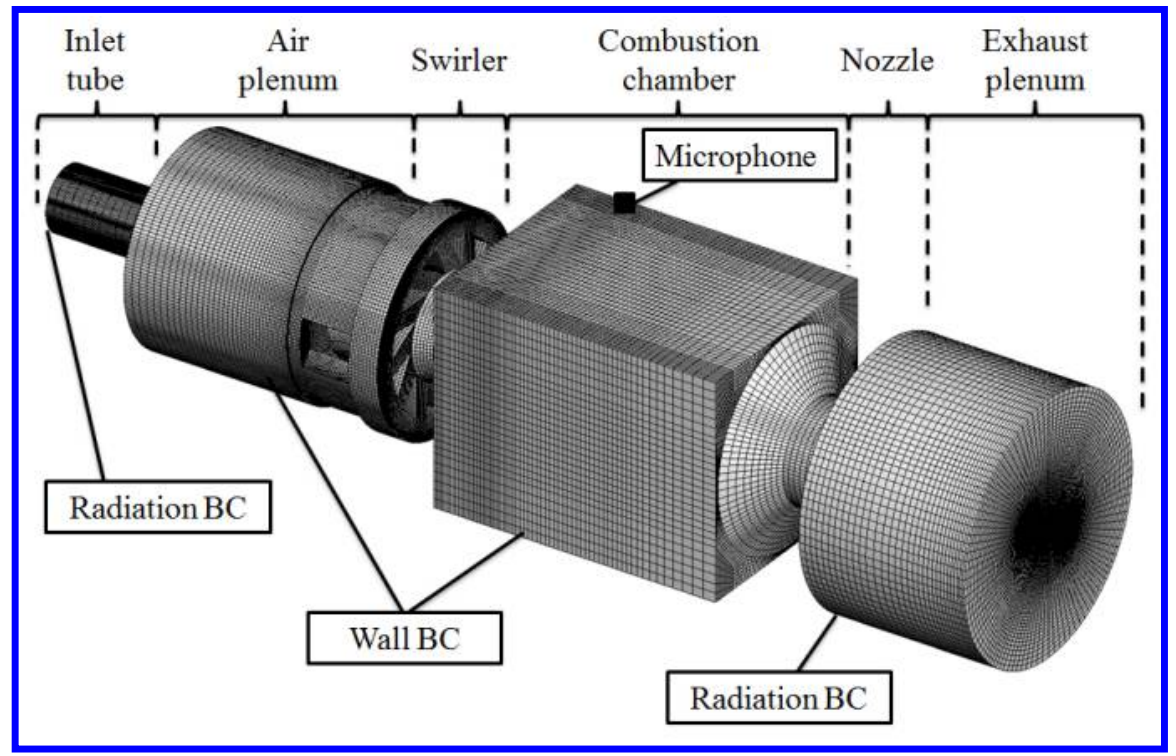

Figure 10: Block-structured computational grid and employed boundary conditions for the combustion acoustics simulations.

The overall simulation time step is limited by the sound propagation modeling and depends on the grid size as well as the local Mach number according to $\Delta t_{\max }=\left(2.83 \Delta x_{\min }\right) /(\pi+\pi M a)$. Therefore, a timestep of $\Delta t=2.2 \cdot 10^{-7} \mathrm{~s}$ is used. The cell determining the maximum time step is located in the combustor outlet tube, where a composition of hot combustion products is present.

The computational domain is enclosed by non-reflecting radiation boundary conditions ${ }^{63}$ at the air plenum inlet tube, a shown in Fig. 10. An additional plenum is attached to the combustor outlet, which is in turn enclosed by a damping sponge layer and radiation conditions. Walls are modeled as fully reflecting, while the ghost point concept of Tam \& Dong ${ }^{64}$ is used.

The spatial extension of the sound source reconstruction domain is determined from the discrete realization of Eq. (17) for no separation time and space. This indicator can be interpreted as the effectively prescribed 
source target variance and is therefore extracted from

$$
\varphi=\frac{\gamma \bar{p}}{\tilde{T}} \sqrt{\frac{\widetilde{T^{\prime \prime 2}}}{\tau_{T}^{2}}} .
$$

The field solution of Eq. (28) based on CFD RANS quantities is shown in Fig. 11, referenced to its maximum value $\varphi / \varphi_{\max }$.

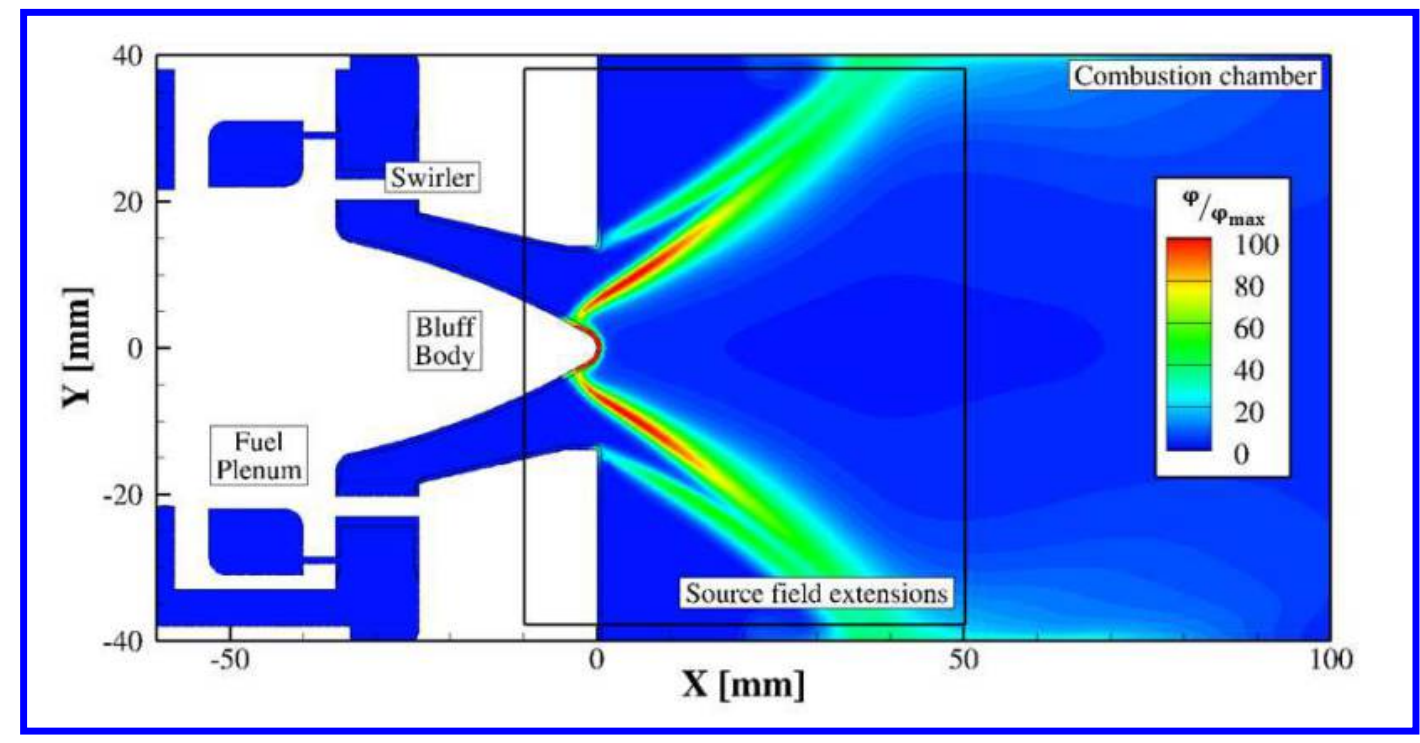

Figure 11: Effectively realized source target standard deviation $\varphi$, normalized to the field maximum value. Evaluated from CFD RANS field solution quantities.

As the main criterion for the determination of source field extensions, field values larger than $5 \%$ of the peak value of $\varphi$ in the inner shear layer are included. This leads to source field extensions of $x \in$ $[-10 \mathrm{~mm} ; 50 \mathrm{~mm}]$ and $y, z \in[-38 \mathrm{~mm} ; 38 \mathrm{~mm}]$. Sources are reconstructed on an auxiliary equidistant and orthogonal grid, with $\Delta=1 \mathrm{~mm}$, which results in $346.6 \mathrm{~K}$ cells and an initial distribution of random particles with 1.154 particles per cell. Sources are obtained via recursive filter ${ }^{51,52}$ operations along the auxiliary grid, accounting for the local integral length scale. Turbulence is synthesized with a first order Langevin approach, as introduced with Eqs. (23) to (25). Integral one-point statistics are extracted from the preceding CFD RANS simulations according to

$$
l_{T}=\frac{\sqrt{k}}{\beta^{*} \omega} \quad \text { and } \quad \tau_{T}=\frac{1}{\beta^{*} \omega} .
$$

A total of $N_{C C A}=9 \cdot 10^{5}$ time steps is simulated, while resulting sound pressure spectra are evaluated over a time span of $\Delta t_{\text {overall }}=0.2 \mathrm{~s}$. Results of the computational combustion acoustics simulations are discussed in the following section.

\section{Computational Combustion Acoustics Results}

Selected results of the acoustics simulations are shown in Fig. 12. Isosurfaces of direct combustion noise sources are superimposed to midplane cuts of the reacting CFD RANS density distribution on the left side. It can be stated that, according to findings in the previous section, direct combustion noise soures in this particular, partially premixed, swirl stabilized case, are mainly located in the inner shear layer in the combustion chamber. Due to the continuously growing lengthscales further downstream, as indicated in Fig. $13 \mathrm{a}$ and Fig. 13b, spatial extensions of sources increase accordingly.

However, largest amplitudes of fluctuation are present rather close to the tip of the flame, as indicated with the effectively realized source variance in Fig. 11. Regarding decorrelation effects of sources, turbulent decay is strongest close to the flame root, where smallest turbulent timescales are present (Fig. 13b). This is reflected in Eq. (27). The first component of the FRPM-inherent correlation function, which is employed for the correlation of combustion noise sources, emphasises this issue: Turbulent timescales grow along the 


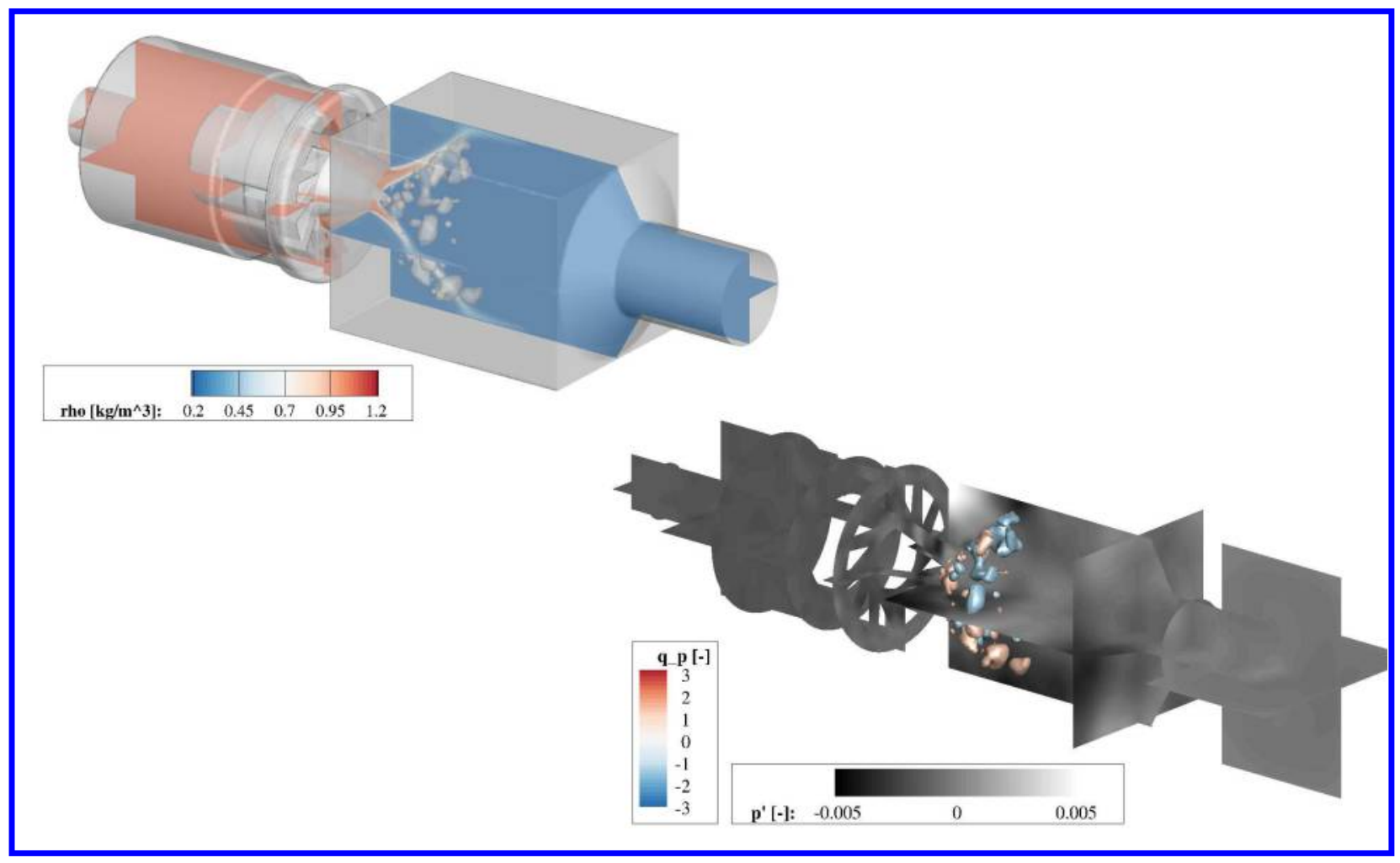

Figure 12: Selected instantaneous combined CFD and CCA results on cuts of combustor planes and exemplary isosurfaces of sound sources. Left: Combustor midplane with density contours from CFD RANS and snapshot of reconstructed combustion noise sources. Right: Slices of instantaneous acoustic pressure $p^{\prime}=p^{\prime} /\left(\rho_{\infty} c_{\infty}^{2}\right)$ and isosurfaces of reconstructed sound sources $q_{p}=q_{p} /\left(f_{r e f} \rho_{\infty} c_{\infty}^{2}\right)$ in the CCA domain.

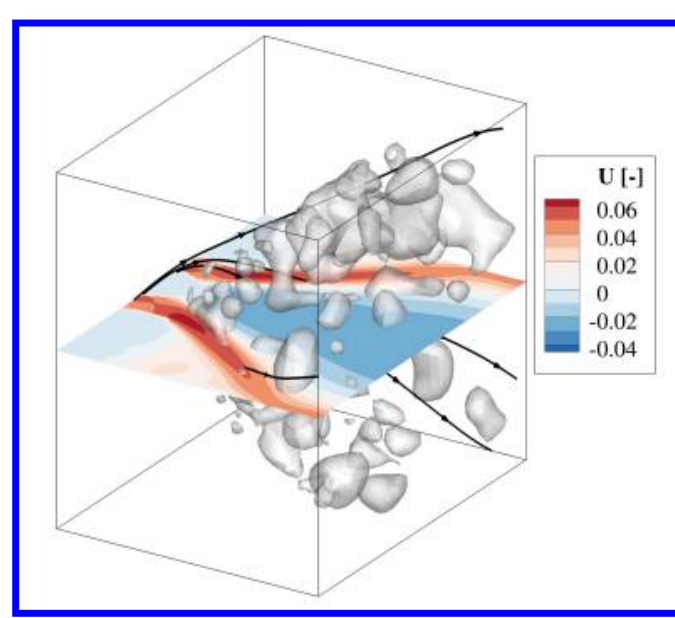

(a) Midplane cut with axial velocity, exemplary streamlines and isosurfaces of direct combustion noise sources.

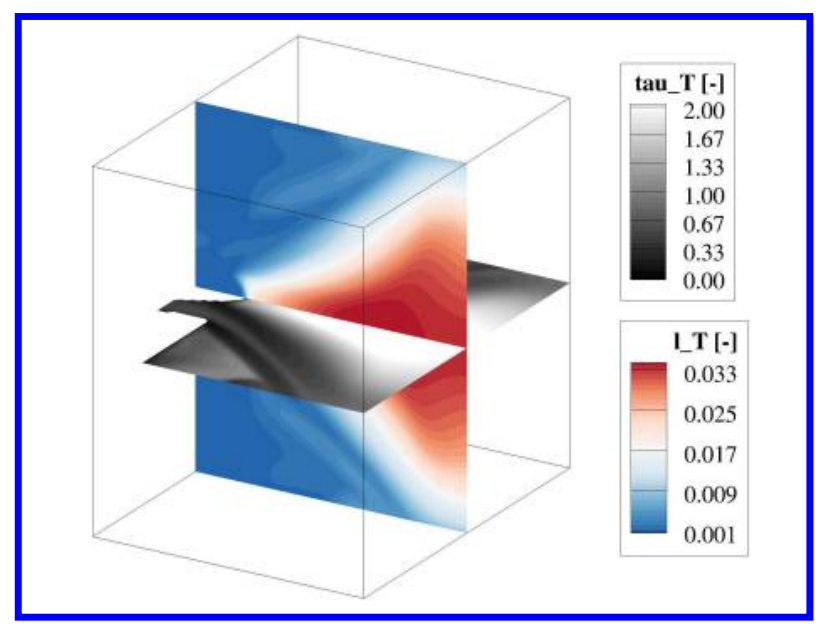

(b) Midplane cuts with integral length- and timescale from reacting CFD RANS simulation.

Figure 13: Source field quantities on midplane cuts and isosurfaces of direct combustion noise sources. 
inner shear layer and therefore downstream decorrelation envelopes of Eq. (27) show a weaker decline. The bottomline is that downstream sound sources have larger spatial extension but smaller fluctuation amplitude in combination with a weaker turbulence induced decay.

An instantaneous field solution of fluctuating acoustic pressure in combination with isosurfaces of direct combustion noise sources is shown in Fig. 12 on the right side. It indicates that pressure fluctuations near the front plate of the combustor are strongest and that no spurious reflection at inlet and outlet radiation boundaries is present. A stable solution of sound propagation with a mean flow field containing large density and flow field gradients is achieved by solving for the modified set of linearized Euler equations, Eq. (18) to Eq. (20). Compared to the genuine linearized Euler equations, components consisting of meanflow gradient terms are neglected, since they are causing instabilities. Therefore, the use of a modified set of basic equations means a compromise between stability of the numerical simulation and the accurate depiction of sound refraction in the combustor.

Pressure spectra of the simulated operation case at the microphone position $x=42.5 \mathrm{~mm}, y=r=42.5 \mathrm{~mm}, z=$ $h=20 \mathrm{~mm}$ are displayed in Fig. 14. Concerning experimental data, two different degrees of premixing of fuel

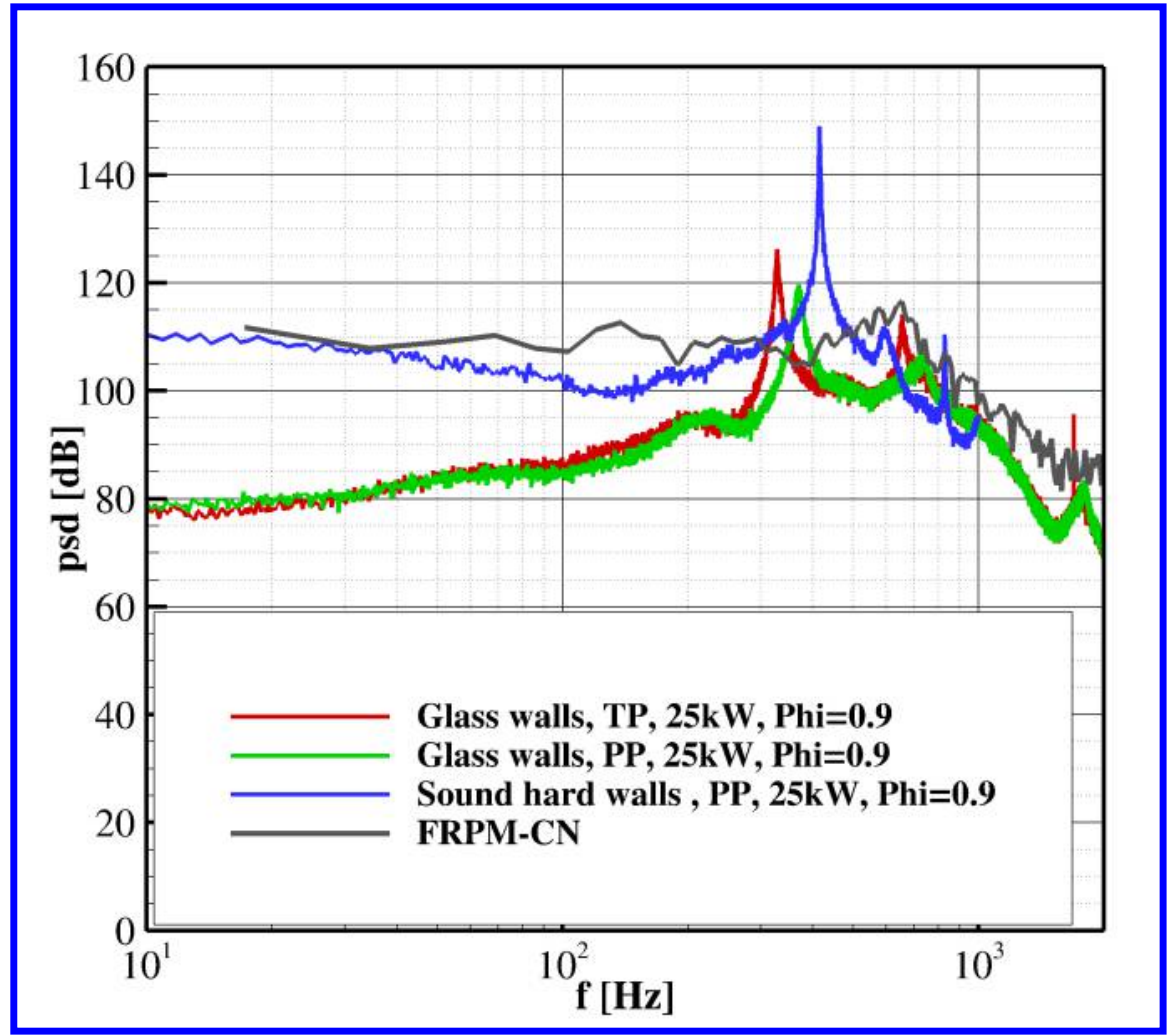

Figure 14: Sound pressure spectra in the combustion chamber at the microphone position $x=42.5 \mathrm{~mm}, y=r=42.5 \mathrm{~mm}, z=h=20 \mathrm{~mm}$. Comparison of experiment and FRPM-CN. TP: technical premixing, PP: perfect premixing.

and air as well as two different boundary conditions for the combustion chamber walls are analyzed. The difference between technical (TP) and perfect (PP) premixing is that in the technical premixing case, fuel is induced through the fuel plenum into the swirler vanes, while for perfect premixing, an air fuel mixture is induced into the upstream air plenum and the fuel plenum has no functionality. As can be seen in Fig. 14, the two cases (red and green curve) show a very similar acoustical behaviour, in terms of peak distribution and absolute sound pressure amplitudes.

The case with perfect premixing is furthermore compared to measurements with sound hard walls. Usually, loosely mounted glass walls for optical access are employed in the combustor, inducing a frequency dependent damping effect. This is estimated here by comparison of measurements of the same operation point but with perfect premixing and sound hard metal walls. The result for comparison of the perfect premixing cases (green and blue curve) is a shift of amplitudes to distinctly higher values, especially for the spectral peaks and levels in the low frequency range. Furthermore, the frequency positions of peaks significantly shift to 
higher values. The technical premixing case is assumed to show similar trends in case of treatment with sound hard wall combustor boundaries.

Experimental data shows several distinct characteristics: Two peaks are present in the spectrum, assigned to an acoustic Helmholtz mode at about $350-400 \mathrm{~Hz}$, while the second peak is assigned to a first harmonic of this phenomenon at $650-800 \mathrm{~Hz}$. A third, less distinct peak at $1700-1800 \mathrm{~Hz}$ indicates a helical flow instability, however, no clear characteristics of such were observed in the experimental investigations. In comparison, the numerical simulation with FRPM-CN has no distinct peak at the respective frequency positions, since thermoacoustic phenomena cannot be depicted with this hybrid, sequential method. However, absolute sound pressure levels are captured very well, especially in the low frequency range, without any artificial scaling in the computational combustion acoustics simulations. The FRPM-CN spectra furthermore show a very similar slope compared to the experimental spectrum in the range of $700-1500 \mathrm{~Hz}$.

The simulations were carried out in a total of 4052 CPU-h, while the CFD fraction of computational time amounts to $1356 \mathrm{CPU}$-h. For comparison with a partially scale resolving simulation of this particular burner, a slightly different operation point was investigated, at $P_{t h}=25.1 \mathrm{~kW}$ and $\Phi=0.7$, which shows a more distinct thermoacoustically unstable behaviour. Those simulations ${ }^{65,66}$ were carried out with the DLR-inhouse code THETA and the fractional step solver SICS, ${ }^{37}$ which allows for the treatment of compressible flows and enables the capturing of acoustics. A detailed combustion model, consisting of a Finite Rate Chemistry (FRC) approach with an assumed PDF ansatz for subgrid scale treatment of turbulence-chemistry interaction was taken into consideration on a detailed DRM19 reaction mechanism. Simulations were conducted on 256 cores in 11 days on an unstructured 44M-cell grid and therefore in $67,584 \mathrm{CPU}$-h in total for the simulation of six mean combustor flow through times or $60 \mathrm{~ms} .{ }^{66}$ FRPM-CN simulation real-time for the evaluation of pressure spectra in the herein treated case is $0.2 \mathrm{~s}$. An equal simulation of realtime with the partially scale resolving method would add up to 225,300 CPU-h. Assuming that similar computational times would be necessary for the simulation of a stable case, FRPM-CN is faster by a factor of approximately 55.6 , meaning one and a half order of magnitudes less computational effort.

\section{Conclusions}

Combustion noise generation and propagation in a laboratory scale burner was simulated with the hybrid, particle based approach FRPM-CN. The partially premixed, swirl stabilized flame was characterized with computationally efficient and robust steady state CFD RANS models and a turbulent combustion noise simulation based on stochastic sound source reconstruction. The treated operation point was selected based on flame shape and sound pressure levels in the combustion chamber, in order to treat a most acoustically stable regime. CFD and CCA (Computational Combustion Acoustics) results were validated with experimental data.

CFD simulations were carried out with the finite-volume based research code THETA by means of a twoequation turbulence model and global treatment of chemical reactions. It was shown that the employed CFD models achieved very good agreement with experimental data, considering flow field and temperature at very low computational cost. A temperature variance field solution was obtained from the solution of an additional transport equation, giving a good representation of experimental profiles.

Consecutively, locations of the main direct combustion noise sources were identified by a discrete realization of the combustion noise source term formulation, based on CFD data. Sound propagation in the acoustics simulations was modeled with a modified set of linearized Euler equations, implemented in the DLR-inhouse code PIANO. ${ }^{62}$ Sound sources were subject to stochastic, particle based reconstruction with local statistics from the preceding CFD RANS simulations as input. Sources were coupled to the linearized basic equations for sound propagation as right hand side monopole forcing of the pressure equation. Mean background flow field quantities were incorporated for the modeling of sound propagation, in order to account for physical sound transportation and refraction.

The comparison of pressure spectra at a combustor microphone position revealed that FRPM-CN reliably predicted absolute sound pressure levels compared to experimentel results at very low computational costs. A comparison of computational turnaround times with a partially scale resolving simulation of a similar operation point demonstrated that FRPM-CN is more than an order of magnitude faster, compared to direct approaches, while FRPM-CN delivers not only acoustics dynamics of the system, but also a true representation of flow field and combustion.

Therefore, FRPM-CN represents a highly robust, easy-to-use and efficient tool not only for research pur- 
poses for industrial applications. Due to the hybrid and sequential character of the method, thermoacoustic dynamics could not be captured, however combustion noise levels were reliably predicted and resonance phenomena are predictable.

\section{References}

${ }^{1}$ ACARE, "Strategic Research Agenda, Volume 1," 2001, www.acare4europe.org.

${ }^{2}$ European Comission, "European Aeronautics: A Vision for 2020," 2001, Office for Official Publications of the European Communities, Luxembourg, ISBN 92-894-0559-7.

${ }^{3}$ European Comission, "Flightpath 2050: Europe's Vision for Aviation," 2011, Office for Official Publications of the European Communities, Luxembourg, ISBN 978-92-79-19724-6, doi 10.2777/5026.

${ }^{4}$ Rolls-Royce, The Jet Engine, Technical Publications Department, Rolls-Royce plc, Derby, England, 1996, ISBN 10: 0902121235.

${ }^{5}$ Deane, E., Use of Fan Rig Data for the Understanding and Prediction of Fan Broadband Noise and Noise Changes due to a Variable Area Nozzle, Dissertation, Institute of Sound and Vibration Research, Faculty of Engineering, Science and Mathematics, University of Southampton, 2009, http://eprints.soton.ac.uk/160877/1.hasCoversheetVersion/P2642.pdf.

${ }^{6}$ Leylekian, L., Lebrun, M., and Lempereur, P., "An Overview of Aircraft Noise Reduction Technologies," Aerospace Lab Journal.

${ }^{7}$ Airbus, "Getting to Grips with Aircraft Noise," 2003.

8 Dowling, A. and Mahmoudi, Y., "Combustion noise," Proceedings of the Combustion Institute, Vol. 35, 2015, pp. 65-100.

${ }^{9}$ Duran, I., Moreau, S., Nicoud, F., Livebardon, T., Bouty, E., and Poinsot, T., "Combustion Noise in Modern AeroEngines," Aerospace Lab Journal, Vol. 7.

${ }^{10}$ Lighthill, M., "On sound generated aerodynamically. I. General Theory," Proceedings of the Royal Society.

${ }^{11}$ Poinsot, T. and Veynante, D., Theoretical and numerical combustion, RT Edwards, Inc., 2005.

$\checkmark{ }^{12}$ Liu, Y., Dowling, A., Swaminathan, N., Morvant, R., Macquisten, M., and Caracciolo, L., "Prediction of Combustion Noise for an Aeroenginge Combustor," Journal of Propulsion and Power, Vol. 30, 2014, pp. 114-122.

${ }^{13}$ Candel, S., Durox, D., Ducruix, S., Birbaud, A.-L., Noiray, N., and Schuller, T., "Flame dynamics and combustion noise: progress and challenges," International Journal of Aeroacoustics, Vol. 8, No. 1, 2009, pp. 1-56.

${ }^{14}$ Mühlbauer, B., Numerische Simulation von Verbrennungslärm, Dissertation, Institut für Verbrennungstechnik der Luftund Raumfahrt, Universität Stuttgart, 2012, http://elib.uni-stuttgart.de/opus/volltexte/2012/7137/.

$\checkmark{ }^{15}$ Bui, T., Schröder, W., and Meinke, M., "Numerical analysis of the acoustic field of reacting flows via acoustic perturbation equations," Computers \& Fluids, Vol. 37, No. 9, 2008, pp. 1157-1169.

${ }^{16}$ Ewert, R. and Emunds, R., "CAA Slat Noise Studies Applying Stochastic Sound Sources Based On Solenoidal Digital Filters," 11th AIAA/CEAS Aeroacoustics Conference, AIAA 2005-2862, 2005.

- ${ }^{17}$ Ewert, R., "Slat Noise Trend Predictions Using CAA With Stochastic Sound Sources From A Random Particle-Mesh Method (RPM)," 12th AIAA/CEAS Aeroacoustics Conference, AIAA 2006-2667, 2006.

${ }^{18}$ Ewert, R., "Broadband Slat Noise Prediction Based on CAA and Stochasic Sound Sources from a Fast Random ParticleMesh (RPM) Method," Computers and Fluids Journal, Vol. 37, No. 4, 2008, pp. 369-387.

$\checkmark{ }^{19}$ Ewert, R., "RPM - the fast Random Particle-Mesh method to realize unsteady turbulent sound sources and velocity fields for CAA applications," 13th AIAA/CEAS Aeroacoustics Conference, AIAA 2007-3506, 2007.

${ }^{20}$ Mühlbauer, B., Ewert, R., Kornow, O., Noll, B., Delfs, J., and Aigner, M., "Simulation of combustion noise using CAA with stochastic sound sources from RANS," 14th AIAA/CEAS Aeroacoustic Conference, 2008, AIAA 2008-2944.

${ }^{21}$ Tam, C. and Auriault, L., "Jet Mixing Noise from Fine-Scale Turbulence," AIAA Journal, Vol. 37, No. 2, 1999, pp. 145153.

${ }^{22}$ Mühlbauer, B., Ewert, R., Kornow, O., and Noll, B., "Evaluation of the RPM Approach for the Simulation of Broadband Combustion Noise," AIAA Journal, Vol. 48, No. 7, 2010, pp. 1379-1390.

${ }^{23}$ Gerlinger, P., Numerische Verbrennungssimulation - Effiziente Numerische Simulation turbulenter Verbrennung, Springer-Verlag, Germany, 2005, ISBN 3-540-23337-7.

- ${ }^{24}$ Ewert, R. and Schröder, W., "Acoustic perturbation equations based on flow decomposition via source filtering," Journal of Computational Physics, Vol. 188, 2003, pp. 365-398.

${ }^{25}$ Mühlbauer, B., Ewert, R., Kornow, O., Boyde, J., Noll, B., Delfs, J., and Aigner, M., "Evaluation of the RPM-CN approach for broadband combustion noise prediction," 15th AIAA/CEAS Aeroacoustic Conference, 2009, AIAA 2009-3285.

${ }^{26}$ Mühlbauer, B., Ewert, R., Kornow, O., Noll, B., and Aigner, M., "Numerical Simulation of Broadband Combustion Noise with the RPM-CN Approach," Proceedings of ASME Turbo Expo 2009, 2009, GT2009-59870.

- ${ }^{27}$ Mühlbauer, B., Ewert, R., Kornow, O., and Noll, B., "Broadband combustion noise simulation of open non-premixed turbulent jet flames," International Journal of Aeroacoustics, Vol. 11, No. 1, 2012, pp. 1-24.

${ }^{28}$ Grimm, F., Ewert, R., Dierke, J., Noll, B., and Aigner, M., "Broadband Combustion Noise Prediction with the Fast Random Particle Method," Proceedings of ASME Turbo Expo 2014, 2014, GT2014-25195.

$\checkmark{ }^{29}$ Ewert, R., Dierke, J., Siebert, J., Neifeld, A., Appel, C., Siefert, M., and Kornow, O., "CAA broadband noise prediction for aeroacoustic design," Journal of Sound and Vibration, Vol. 330, No. 17, 2011, pp. 4139-4160.

${ }^{30}$ Grimm, F., Ewert, R., Dierke, J., Noll, B., and Aigner, M., "The Fast Random Particle Method for Combustion Noise Prediction," 20th AIAA/CEAS Aeroacoustics Conference, 2014, AIAA Paper 2014-2451.

${ }^{31}$ Grimm, F., Ewert, R., Dierke, J., Noll, B., and Aigner, M., "Efficient Full 3D Turbulent Combustion Noise Simulation Based on Stochastic Sound Sources," 21st AIAA/CEAS Aeroacoustics Conference, 2015, AIAA Paper 2015-2973. 
-32 Ewert, R., Neifeld, A., and Fritzsch, A., "A 3-D modal stochastic jet noise source model," 17th AIAA/CEAS Aeroacoustics Conference, 2011, AIAA Paper 2011-2887.

${ }^{33}$ Domenico, M. D., Numerical simulations of soot formation in turbulent flows, Dissertation, Institut für Verbrennungstechnik der Luft- und Raumfahrt, Universität Stuttgart, 2008, http://elib.uni-stuttgart.de/opus/volltexte/2008/3624/.

${ }^{34}$ Reichling, G., Noll, B., and Aigner, M., "Development of a Projection-Based Method for the Numerical Calculation of Compressible Reactive Flows," Proceedings of the 51st AIAA Aerospace Sciences Meeting including the New Horizons Forum and Aerospace Exposition, 2013, AIAA 2013-1003.

${ }^{35}$ Reichling, G., Noll, B., and Aigner, M., "Numerical Simulation of the Non-Reactive and Reactive Flow in a Swirled Model Gas Turbine Combustor," 21st AIAA Computational Fluid Dynamics Conference, 2013, AIAA 2013-2434.

${ }^{36}$ Reichling, G., Development of Numerical Methods for the Calculation of Thermo-Acoustic Interactions in Gas Turbine Combustion Chambers, Dissertation, Institut für Verbrennungstechnik der Luft- und Raumfahrt, Universität Stuttgart, 2015, http://elib.uni-stuttgart.de/opus/volltexte/2015/9922/.

${ }^{37}$ Lourier, J.-M., Noll, B., and Aigner, M., "Extension of a Compressible Pressure-Based Solver for Reacting Flows," 19th AIAA/CEAS Aeroacoustics Conference, 2013, AIAA 2013-2101.

${ }^{38}$ Lourier, J.-M., Numerische Simulation von thermoakustischen Instabilitten in Gasturbinenbrennkammern mithilfe von Impedanzrandbedingungen, Dissertation, Institut für Verbrennungstechnik der Luft- und Raumfahrt, Universität Stuttgart, 2015.

${ }^{39}$ Wilcox, D., Turbulence modeling for CFD, La Canada, California : DCW Industries Inc., 2006, 3rd Edition, ISBN 978-1-928729-08-2.

${ }^{40}$ Ivanova, E., Numerical Simulations of Turbulent Mixing in Complex Flows, Dissertation, Institut für Verbrennungstechnik der Luft- und Raumfahrt, Universität Stuttgart, 2012, http://elib.uni-stuttgart.de/opus/volltexte/2013/7813/.

${ }^{41}$ Boussinesq, J., "Essai sur la theorie des eaux courantes," Memoires presentes par divers savants a l'Academie des Sciences, Vol. 23, No. 1, 1877, pp. 1-680.

${ }^{42}$ Menter, F., Kuntz, M., and Langtry, R., "Ten Years of Industrial Experience with the SST Turbulence Model," Turbulence, Heat and Mass Transfer 4, ed: K. Hanjalic, Y. Nagano, and M. Tummers, Begell House, Inc., 2003, pp. 625-632.

43 Jones, W. and Launder, B., "The prediction of laminarization with a two-equation model of turbulence," International Journal of Heat and Mass Transfer, Vol. 15, No. 2, 1972, pp. 301-314.

${ }^{44}$ Launder, B. and Sharma, B., "Application of the energy-dissipation model of turbulence to the calculation of flow near a spinning disc," Letters in Heat and Mass Transfer, Vol. 1, 1974, pp. 131-138.

${ }^{45}$ Wilcox, D., "Reassessment of the scale-determining equation for advanced turbulence models," AIAA Journal, Vol. 26, 1988, pp. 1299-1310.

${ }^{46}$ Magnussen, B. and Hjertager, B., "On Mathematical Modelling of Turbulent Combustion with Special Emphasis on Soot Formation and Combustion," 16th Symposium (International) on Combustion, Vol. 16, No. 1, 1977, pp. 719-729.

${ }^{47}$ Magnussen, B., "On the structure of turbulence and a generalized eddy dissipation concept for chemical reaction in turbulent flow," 19th AIAA Aerospace Science Meeting, 1981, AIAA 1981-42.

${ }^{48}$ Warnatz, J., Maas, U., and Dibble, R., Combustion: physical and chemical fundamentals, modeling and simulation, experiments, pollutant formation, Springer, 2006.

${ }^{49}$ Joos, F., Technische Verbrennung: Verbrennungstechnik, Verbrennungsmodellierung, Emissionen, Springer Verlag Berlin Heidelberg, ISBN: 978-3-540-34333-2, 2006.

${ }^{50}$ Ewert, R., Kornow, O., Delfs, J., Yin, J., Röber, T., and Rose, M., "A CAA Based Approach to Tone Haystacking," 15th AIAA/CEAS Aeroacoustics Conference, AIAA 2009-3217, 2009.

$\checkmark{ }^{51}$ Purser, R., Wu, W.-S., Parrish, D., and Roberts, N., "Numerical aspects of the application of recursive filters to variational statistical analysis. part i: Spatially homogeneous and isotropic gaussian covariances," Monthly Weather Review, Vol. 131, 2003, pp. 1524-1535.

52 Purser, R., Wu, W.-S., Parrish, D., and Roberts, N., "Numerical aspects of the application of recursive filters to variational statistical analysis. part ii: Spatially inhomogeneous and anisotropic gaussian covariances," Monthly Weather Review, Vol. 131, 2003, pp. 1536-1548.

${ }^{53}$ Dem, C., Stöhr, M., Arndt, C., Steinberg, A., and Meier, W., "Experimental study of turbulence-chemistry interactions in confined swirl flames with different degrees of premixing," Zeitschrift für Physikalische Chemie.

${ }^{54}$ Oberleithner, K., Stöhr, M., Im, S., Arndt, C., and Steinberg, A., "Formation and flame-induced suppression of the precessing vortex core in a swirl combustor: Experiments and linear stability analysis," Combustion and Flame, Vol. 162, No. 8, 2015, pp. 3100-3114.

${ }^{55}$ Meier, W., Weigand, P., Duan, X., and Giezendanner-Thoben, R., "Detailed characterization of the dynamics of thermoacoustic pulsations in a lean premixed swirl flame," Combustion and Flame, Vol. 150, 2007, pp. 2-26.

${ }^{56}$ Werner, S. and Stöhr, M., Personal communication, 2015.

${ }^{57} \mathrm{Im}, \mathrm{S} .$, Caractrisation des instabilits thermoacoustiques dans les chambres de combustion gaz, Master Thesis, German Aerospace Center and Ecole Centrale Paris, 2013, Supervisor: Dr. M. Stöhr and Dr. W. Meier.

${ }^{58}$ Domenico, M. D., "Private Communication," 2015.

${ }^{59}$ Domenico, M. D., Gerlinger, P., and Noll, B., "Numerical Simulations of Confined, Turbulent, Lean, Premixed Flames using a Detailed Chemistry Combustion Model," Proceedings of the ASME Turbo Expo 2011, 2011, GT2011-45520.

-60 Westbrook, C. and Dryer, F., "Simplified Reaction Mechanisms for the Oxidation of Hydrocarbon Fuels in Flames," Combustion Science and Technology, Vol. 27, 1981, pp. 31-43.

${ }^{61}$ Krige, D., "A statistical approach to some basic mine valuation problems on the Witwatersrand," J. of the Chem., Metal and Mining Soc. of South Africa, Vol. 52, No. 6, 1951, pp. 119-139.

${ }^{62}$ Delfs, J., Bauer, M., Ewert, R., Grogger, H., Lummer, M., and Lauke, T., Numerical Simulation of Aerodynamic Noise with DLR's aeroacoustic code PIANO, DLR - German Aerospace Center, Braunschweig, Germany, 5th ed., 2008. 
-63 Tam, C. and Webb, J., "Dispersion-Relation-Preserving Finite Difference Schemes for Computational Acoustics," Journal of Computational Physics, Vol. 107, No. 2, 1993, pp. 262-281.

${ }^{64}$ Tam, C. and Dong, Z., "Wall boundary conditions for high-order finite-difference schemes in computational aeroacoustics," Theoret. Comput. Fluid Dynamics, Vol. 6, 1994, pp. 303-322.

${ }^{65}$ Lourier, J.-M., Noll, B., and Aigner, M., "Large Eddy Simulation of a Thermoacoustic Instability within a Swirl-Stabilized Burner using Impedance Boundary Conditions," Proceedings of ASME Turbo Expo 2014, 2014, GT2014-26200.

${ }^{66}$ Lourier, J.-M., Eberle, C., Noll, B., and Aigner, M., "Influence of Turbulence-Chemistry Interaction Modeling on the Structure and the Stability of a Swirl-Stabilized Flame," Proceedings of ASME Turbo Expo 2015, 2015, GT2015-43174. 